ARTICLE

https://doi.org/10.1038/s41467-019-11636-5

\title{
Mul1 restrains Parkin-mediated mitophagy in mature neurons by maintaining ER-mitochondrial contacts
}

\author{
Rajat Puri ${ }^{1}$, Xiu-Tang Cheng (D) ${ }^{1}$, Mei-Yao Lin ${ }^{1}$, Ning Huang (D) $^{1} \&$ Zu-Hang Sheng (D ${ }^{1}$
}

Chronic mitochondrial stress associates with major neurodegenerative diseases. Recovering stressed mitochondria constitutes a critical step of mitochondrial quality control and thus energy maintenance in early stages of neurodegeneration. Here, we reveal Mul1-Mfn2 pathway that maintains neuronal mitochondrial integrity under stress conditions. Mul1 deficiency increases Mfn2 activity that triggers the first phasic mitochondrial hyperfusion and also acts as an ER-Mito tethering antagonist. Reduced ER-Mito coupling leads to increased cytoplasmic $\mathrm{Ca}^{2+}$ load that activates calcineurin and induces the second phasic Drp1dependent mitochondrial fragmentation and mitophagy. Overexpressing Mfn2, but not Mfn1, mimics Mul1-deficient phenotypes, while expressing PTPIP51, an ER-Mito anchoring protein, suppresses Parkin-mediated mitophagy. Thus, by regulating mitochondrial morphology and ER-Mito contacts, Mul1-Mfn2 pathway plays an early checkpoint role in maintaining mitochondrial integrity. Our study provides new mechanistic insights into neuronal mitochondrial maintenance under stress conditions, which is relevant to several major neurodegenerative diseases associated with mitochondrial dysfunction and altered ER-Mito interplay.

\footnotetext{
${ }^{1}$ Synaptic Function Section, The Porter Neuroscience Research Center, National Institute of Neurological Disorders and Stroke, National Institutes of Health, Room 2B-215, 35 Convent Drive, Bethesda, MD 20892-3706, USA. Correspondence and requests for materials should be addressed to Z.-H.S. (email: shengz@ninds.nih.gov)
} 
M itochondria supply ATP required for neuronal growth, survival, and function. Mitochondrial dysfunction and energy deficits are linked to major neurodegenerative diseases $^{1-3}$. Mitophagy, a key pathway to eliminate damaged mitochondria ${ }^{4}$, is mediated by Parkinson's disease (PD) genes PTEN-induced kinase 1 (PINK1; PARK6) and cytosolic E3 ubiquitin ligase Parkin (PARK2). Following mitochondrial depolarization, PINK1 is stabilized on the outer mitochondrial membrane (OMM), which leads to recruitment of cytosolic Parkin, triggering the ubiquitination of OMM proteins and the engulfment and degradation of damaged mitochondria through the lysosomal system. As neurons are post-mitotic cells that survive for the lifetime of the organism, recovery rather than acute degradation of stressed mitochondria is advantageous for mitochondrial maintenance and energy homeostasis.

Our previous study in mature cortical neurons revealed that mitophagy is observed in a small proportion of neurons following mitochondrial depolarization and that Parkin translocation to depolarized mitochondria occurs much more slowly than in nonneuronal cells ${ }^{5,6}$. Consistently, mitophagy is observed in a small proportion of axonal mitochondria following acute depolarization ${ }^{7}$. Parkin or PINK1 knock-out mice showed subtle changes in mitochondrial morphology and neuronal degeneration ${ }^{8-10}$. In parkin and pink mutant flies, the density and integrity of axonal mitochondria in motor neurons is comparable to WT controls ${ }^{11,12}$. These findings argue for intrinsic neuronal mechanisms that can maintain or recover mitochondrial integrity, rather than rapid elimination of dysfunctional mitochondria through Parkin-mediated mitophagy. To support this assumption, several fundamental questions remain to be addressed: (1) Do distinct mechanisms are in place in neurons to act as a checkpoint for recovery vs. rapid degradation of chronically stressed mitochondria? (2) Is mitophagy the second resort for neuronal mitochondrial quality control after recovery mechanisms have failed? (3) If this is the case, does mitochondrial ubiquitin ligase 1 (Mul1) play an early role in maintaining neuronal mitochondrial integrity? Addressing these questions is relevant to major neurodegenerative diseases associated with chronic mitochondrial stress.

Mul1, also referred to as mitochondrial-anchored protein ligase $(\mathrm{MAPL})^{13}$, mitochondrial ubiquitin ligase activator of NF- $\mathrm{kB}$ (MULAN) ${ }^{14}$, or growth inhibition and death E3 ligase (GIDE) ${ }^{15}$, is a multifunctional mitochondrial membrane protein. In nonneuronal cell lines, Mul1 acts as an E3 ubiquitin ligase that binds, ubiquitinates, and degrades $\mathrm{Mfn} 2^{16}$ and as a small ubiquitin-like modifier (SUMO) E3 ligase that SUMOylates dynamin-related protein-1 (Drp1) to enhance its stability on mitochondria ${ }^{17,18}$. Mfn2 is known to control mitochondrial fusion and regulate the interplay between the endoplasmic reticulum and mitochondria (ER-Mito) ${ }^{19-21}$ in mouse embryonic fibroblasts (MEFs) and Hela cells. ER-Mito contacts are tethered by multiple linker proteins with diverse distribution and interactions between the two organelles ${ }^{22}$. Among these linker proteins is tyrosine phosphatase interacting protein 51 (PTPIP51), which enhances ER-Mito interaction $^{23,24}$. ER-Mito contacts maintain lipid and energy metabolism, as well as $\mathrm{Ca}^{2+}$ transfer from the ER to mitochondria that is essential for mitochondrial bioenergetics and integrity 25,26 . The ER extends from the soma into dendrites and axons $^{27}$, thus allowing for distal ER-Mito signaling ${ }^{28}$. Disrupted ER-Mito contacts have been implicated in several major neurodegenerative diseases ${ }^{23,29,30}$.

PINK1 or Parkin null mutants in Drosophila show striking mitochondrial phenotypes ${ }^{31}$; these phenotypes are strongly suppressed by overexpressing Mul1 ${ }^{16}$. Loss of Mull leads to increased Mfn1 and Mfn2 levels in HeLa cells ${ }^{16}$. Given the fact that Parkin-mediated mitophagy is significantly delayed in mature primary neurons in response to chronic mitochondrial stress under physiological and pathological conditions, we hypothesize that neurons have an intrinsic mechanism for recovering chronically stressed mitochondria through the mitochondria-resident Mul1-Mfn pathway before recruiting cytosolic Parkin to damaged mitochondria. If this checkpoint mechanism fails, Parkin-mediated mitophagy is then activated. Here, by testing this hypothesis we reveal that the Mul1-Mfn2 pathway maintains neuronal mitochondrial integrity through its dual-role in regulating mitochondrial morphology and ER-Mito contacts. This mechanism ensures that mitophagy degradation is restrained in neurons under early stress conditions. Identifying this pathway is particularly relevant because chronical mitochondrial dysfunction and altered ER-Mito contacts have been reported in Alzheimer's disease (AD), PD, amyotrophic lateral sclerosis (ALS), and hereditary spastic paraplegia (HSP) $23,32,33$.

\section{Results}

Mul1 protects neuronal mitochondria from mitophagy. While the PINK1-Parkin pathway in eliminating dysfunctional mitochondria has been well characterized in many cell types ${ }^{34}$, primary neurons often show delayed Parkin-mediated mitophagy in response to the depolarization of mitochondrial membrane potential $\left(\Delta \psi_{m}\right)^{5,6,35}$. These studies raise the question of whether an alternative pathway acts as the first-line of surveillance that maintains or recovers neuronal mitochondrial integrity before activating mitophagy. Our hypothesis is that mitochondrial E3 ubiquitin ligase Mull plays an early role in maintaining neuronal mitochondrial integrity following mild stress before cytosolic E3 ubiquitin ligase Parkin is recruited to mitochondria for mitophagy. To test our hypothesis, we established a Mul1-deficient cortical neuron system by knocking down Mull expression with Mul1-targeted short hairpin RNA (Mul1-shRNA) or a scrambled hairpin RNA as a control (scr-shRNA); both were characterized in the previous study ${ }^{16}$. Mul1, but not mitochondrial proteins Hsp60 and Tom20, was largely suppressed in cells expressing Mul1-shRNA (Supplementary Fig. 1a). Mull has two mitochondrial transmembrane segments (TM1, TM2) and a RING finger domain with E3 ubiquitin ligase activity at the C-terminus. To generate Mul1-deficient cortical neurons, we expressed RINGdeleted Mul1 mutant (Mul1 $\Delta$ Ring) or a single residue mutation in RING (Mul1-H319A). As an off mitochondria-targeting control, we also expressed a truncated Mull by deleting both TM1 and TM2 (Mul1 $\Delta$ TM1/2) (Supplementary Fig. 1b). WT and mutant Mul1 (Mul1 $\Delta$ Ring and Mul1-H319A) are targeted to neuronal mitochondria labeled with cytochrome $\mathrm{c}$ or DsRedMito; Mul1 $\Delta \mathrm{TM} 1 / 2$ displays diffused distribution in neurons at days in vitro 10-11 (DIV10-11) (Supplementary Fig. 1c, d).

We asked whether Mul1 deficiency facilitates recruitment of cytosolic Parkin to stressed mitochondria in neurons. Acute mitochondrial depolarization with a high dose $(40 \mu \mathrm{M})$ of antimycin A (AA), a respiratory complex III inhibitor, rapidly induces Parkin-mediated mitophagy in $\sim 50 \mathrm{~min}^{7}$. To study the mitochondrial quality control in response to mild stress, we instead used $100 \mathrm{nM}$ of AA, a 400-time lower dose than widely used in the literature ${ }^{7,36}$. We previously tested the effect of different dose of AA on mitochondrial integrity by measuring mitochondrial oxygen consumption rate (OCR) in cortical neurons ${ }^{6}$. Nanomolar dose AA induces mild mitochondrial stress relevant to chronic mitochondrial dysfunction associated with neurodegenerative diseases; this treatment is able to maintain neuronal survival, thus allowing us to perform various live imaging and cell biology studies. We measured $\Delta \psi_{\mathrm{m}}$ with the cellpermeant, red-orange fluorescent cationic dye tetramethylrhodamine ethylester (TMRE), which labels polarized mitochondria 
with an inner $\Delta \psi_{\mathrm{m}}$, following a 3-h treatment with $100 \mathrm{nM}$ AA or DMSO control. This mild AA treatment is sufficient to depolarize mitochondria (Supplementary Fig. 2), allowing our study of mitochondrial maintenance.

To examine whether Mul1 deficiency facilitates mitophagy, we measured the percentage of neurons displaying Parkin translocation under a mild depolarization condition ${ }^{6}$. Cortical neurons at DIV8 were co-transfected with mCherry-Parkin and CFP-Mito, together with GFP-Mul1, GFP-Mul1 $\triangle$ Ring, scr-shRNA, or Mul1shRNA, followed by incubation at DIV10 with $100 \mathrm{nM}$ AA for $3 \mathrm{~h}$ before fixation and imaging. Mild depolarization triggers Parkin translocation in a small portion of neurons expressing scr-shRNA or GFP-Mul1. In contrast, Mull deficiency induces more neurons to recruit Parkin into stressed mitochondria (Fig. 1a-e, Supplementary Fig. 2c). The average percentage of neurons displaying Parkin recruitment is $37.06 \pm 2.47 \%$ (Mul1-shRNA, $p<0.001$, Dunnett's post hoc test) and $24.77 \pm 2.21 \%$ (Mul1 $\Delta$ Ring, $p<$ $0.01)$, respectively, relative to the baseline of neurons expressing GFP $(14.84 \pm 1.56 \%)$ following mild stress. Conversely, elevated Mul1 expression suppresses Parkin translocation (5.54 $\pm 0.67 \%$, $p<0.05)$. Analysis of Pearson's correlation coefficient indicates a correlation of Parkin signal on mitochondria in Mull-shRNA neurons (Fig. 1f). Even in the absence of AA, Mull deficiency is sufficient to induce mitophagy in neurons, where fragmented mitochondria are readily observed; some of which are subjected to autophagic clearance by recruiting the autophagic markers p62 and LC3 (Supplementary Fig. 3) or sorted into lysosomes (Fig. 1g).

To further confirm enhanced mitophagy in Mull-deficient neurons, we alternatively measured dynamic mitophagy using $\mathrm{mt}$-Keima, a ratiometric $\mathrm{pH}$-sensitive fluorescent probe that targets mitochondrial matrix ${ }^{37}$. Keima has an excitation spectrum that changes according to $\mathrm{pH}$ : a short wavelength $(458 \mathrm{~nm})$ is predominant for excitation in a neutral environment, whereas a long wavelength $(561 \mathrm{~nm})$ is predominant in an acidic lysosomal environment. When mitochondria are healthy, mt-Keima displays a low fluorescence ratio $(561 \mathrm{~nm} / 458 \mathrm{~nm}$ ) (lysosomal signal $\mathrm{red} /$ mitochondrial signal green); however, when mitochondria are engulfed by acidic lysosomes, mt-Keima display a high fluorescence ratio (stronger red signal). Since mt-Keima is resistant to lysosomal proteases, it allows for measurement of dynamic and cumulative mitophagy processes. Analysis of "mitophagy index" by measuring relative ratio of $561 \mathrm{~nm} / 458 \mathrm{~nm}$ in the cell body supports our notion that Mull deficiency enhances dynamic mitophagy in neurons with Mull depletion or overexpression of Mul1 $\Delta$ Ring (Fig. 1h, i). Altogether, these data suggest an essential role for Mull in suppressing neuronal mitophagy under mild stress conditions.

Mul1 deficiency triggers biphasic mitochondrial dynamics. Given that overexpression or depletion of Mul1 in non-neuronal cells perturbs mitochondrial dynamics ${ }^{13,14}$ and Mul1 promotes ubiquitin-dependent degradation of Mfn through its E3 ubiquitin ligase activity ${ }^{16}$, we asked whether Mull maintains neuronal mitochondrial morphology. By knocking down Mul1 in cortical neurons with Mul1-shRNA, we observed a striking mitochondrial phenotype: a biphasic transition from hyperfusion at DIV10-11 to fragmentation at DIV14-15. Such phenotypes are not observed in control neurons with scr-shRNA (Fig. 2a). We characterized the morphological transition by measuring the average mitochondrial size (area) and shape (aspect ratio of major/minor axis) at twotime points (DIV10-11 and DIV14-15) in both dendrites and axons. Elongated tubular mitochondria (arrows) at DIV10-11 and fragmented mitochondria (arrowheads) at DIV14-15 in both dendrites (Fig. 2b, c) and axons (Supplementary Fig. 4a) were readily observed in Mull-deficient neurons transfected with Mul1-shRNA, Mul1 $\Delta$ Ring, or Mul1-H319A. Such phenotypes were rarely detected in control neurons expressing GFP or scrshRNA. At DIV10-11, the average size of dendritic mitochondria in Mul1-deficient neurons varies from $\geq 4 \mu \mathrm{m}^{2}$ (Mul1 $\Delta$ Ring, Mul1-H319A) to $>5 \mu \mathrm{m}^{2}$ (Mul1-shRNA) compared with $\leq 3 \mu \mathrm{m}^{2}$ for control neurons (GFP or scr-shRNA). Increased size of dendritic mitochondria is attributed to mitochondrial elongation as indicated by an increase in the aspect ratio of $>8$ (Mul1-shRNA) or $>6$ for (Mul1 $\Delta$ Ring, Mul1-H319A) compared with $<5$ (GFP or sc-shRNA). Consistently, frequency distributions of mitochondrial size and aspect ratio show elongated mitochondria at DIV10-11 in both dendrites and axons in Mul1-deficient neurons (Supplementary Fig. 4b, c). However, the hyperfusion status (Phase I) is only maintained a few days; tubular mitochondria are quickly transitioned into fragmentation (Phase II) at DIV14-15. Mitochondrial fragmentation in Mull-deficient neurons is mostly striking in dendrites, where mitochondria have an average size of $\leq 1 \mu \mathrm{m}^{2}$ (Mul1-shRNA, Mul1 $\Delta$ Ring) to $\leq 2.5 \mu \mathrm{m}^{2}$ (Mul1-H319A) and an aspect ratio ranging from $<3$ (Mul1 shRNA, Mul1 $\Delta$ Ring) to $<4$ (Mul1-H319A) (Fig. 2c, d). Knockdown of Mul1 showed more robust fragmentation than expressing mutant Mul1. Control neurons expressing GFP or scr-shRNA showed no detectable change in mitochondrial morphology, supporting our notion that Mul1 deficiency induces mitochondrial hyperfusion at phase I and fragmentation at phase II. While overexpressing Mul1 did not induce the biphasic change in mitochondrial morphology (Fig. 2b-d), fragmentation in Mull-deficient neurons was partially reversed by re-introducing GFP-Mul1 (Supplementary Fig 4d).

To confirm the specificity of Mul1-shRNA, we generated an shRNA-resistant Mull mutant $\left(\mathrm{Mul1}^{\star}\right)$ by substituting eight third-code nucleotides in shRNA-targeting sequence without changing the amino acid. While Mull-shRNA suppressed endogenous Mull and significantly depleted exogenous FlagMul1 ( $p<0.001$, Kruskal-Wallis-Test), it failed to suppress Mul1* $(p=0.68)$, thus indicating an shRNA-resistant Mull mutant (Supplementary Fig. 5a, b). Mitochondrial fragmentation and depolarization in Mul1-deficient neurons (Mul1-shRNA) can be effectively rescued by co-expressing Mul1* (Supplementary Fig. $5 c-f)$. The rescue of two key Mul1-depleted phenotypes indicates the specificity of Mul1-shRNA, thus excluding an offtarget effect.

Fragmented mitochondria lose integrity and ATP production. We next asked whether the biphasic change in mitochondrial morphology is associated with altered mitochondrial integrity. We examined $\Delta \psi_{\mathrm{m}}$ at DIV10-11 and DIV14-15, two-time points associated with hyperfusion and fragmentation, respectively. Mitochondria were labeled by CFP-Mito (Fig. 3a-d), GFP-Mul1H319A (Fig. 3e), or GFP-Mul1 (Fig. 3f). Live neurons at both DIV10-11 and DIV14-15 were loaded with TMRE. TMRE intensity was measured in somato-dendritic mitochondria. Control neurons expressing GFP, scr-shRNA + GFP, or GFP-Mul1 display polarized mitochondria at DIV10-11 and DIV14-15. While TMRE intensity is maintained at DIV10-11 in Mul1deficient neurons, it is significantly reduced at DIV14-15 (Fig. 3g), suggesting that hyperfused mitochondria are able to maintain their $\Delta \psi_{\mathrm{m}}$, whereas fragmented ones largely lose their $\Delta \psi_{\mathrm{m}}$.

Mitochondria ATP production depends upon the maintenance of $\Delta \psi_{\mathrm{m}}$. To assess ATP production capacity in Mul1-deficient neurons, we measured cellular ATP levels using a genetically encoded ratiometric FRET-based ATP sensor (GoAteam2) ${ }^{38}$, as we previously reported ${ }^{39}$. Neurons were co-transfected with 
a

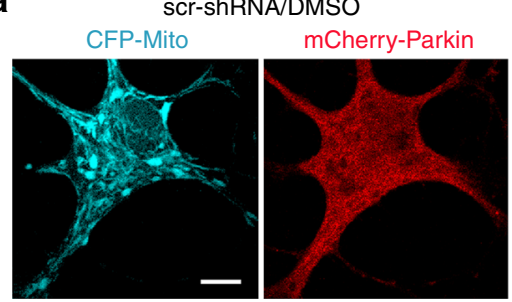

b

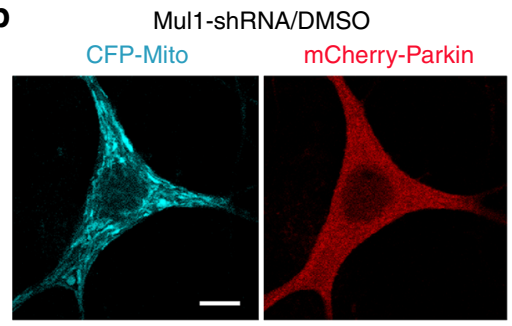

C

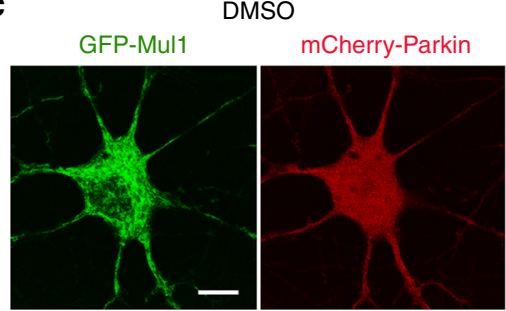

d

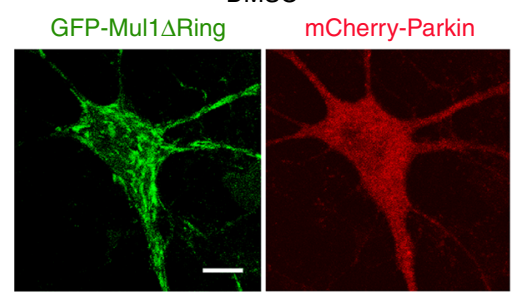

g

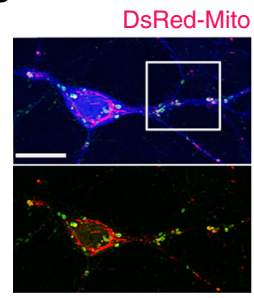

Mul1-shRNA
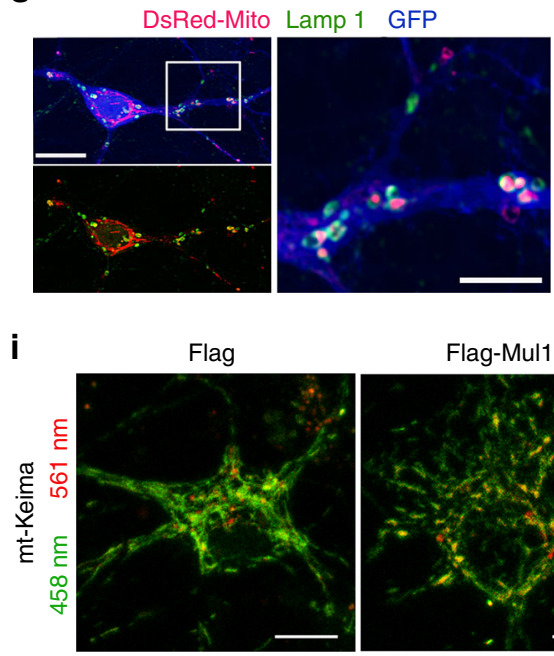
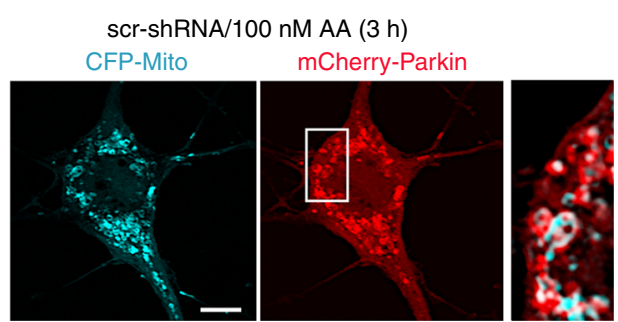

e
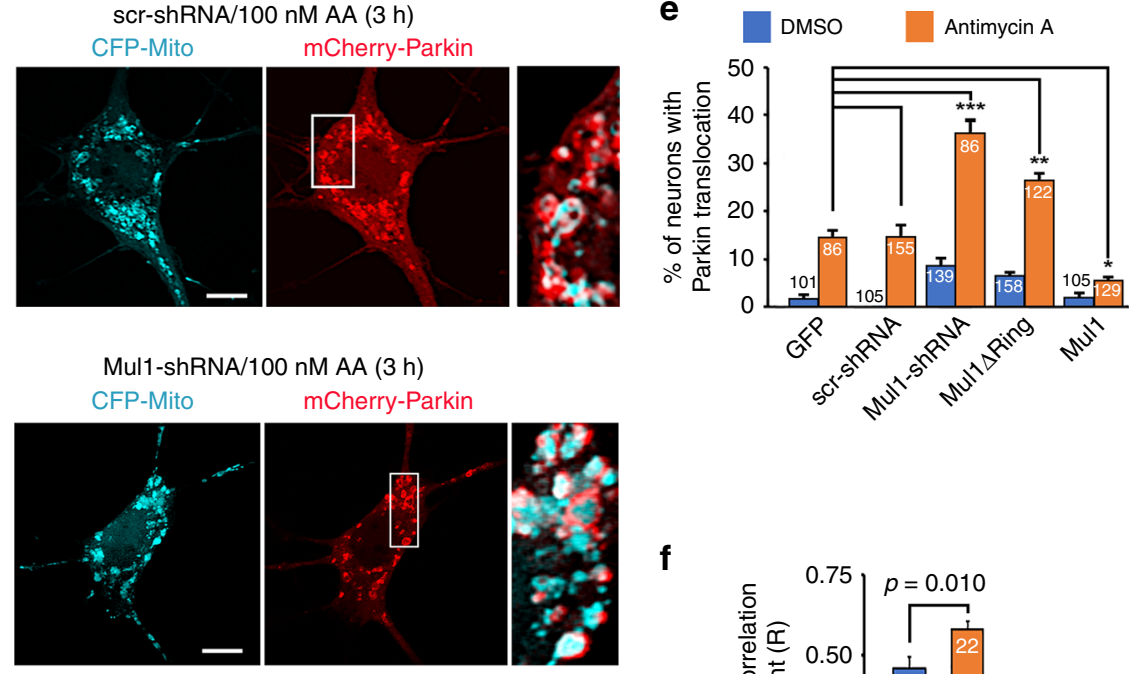

$100 \mathrm{nM}$ AA (3 h)

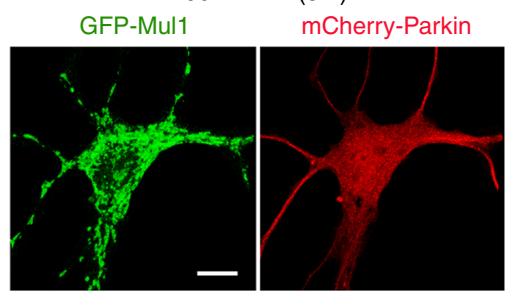

$100 \mathrm{nM}$ AA $(3 \mathrm{~h})$

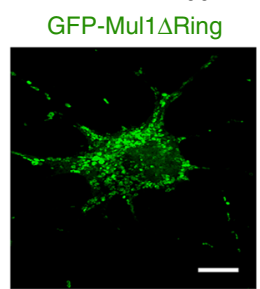

mCherry-Parkin
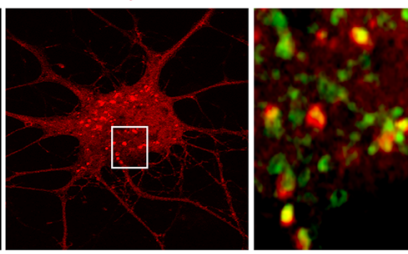

f

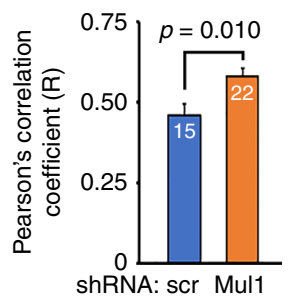

h
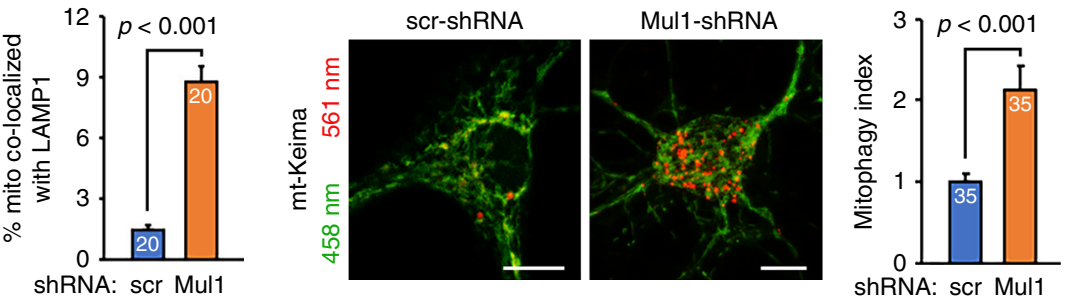

shRNA: scr Mul1
GoAteam2 and Mul1 or Mul1 $\Delta$ Ring at DIV7-8. The relative ATP levels were measured by analysis of the emission ratio (560/510) when excited with a 458-nm laser line at both DIV10-11 and DIV14-15. A significant reduction in cellular ATP levels was detected in neurons expressing Mul1 $\Delta$ Ring in the soma-dendritic region, dendrites, and axons (Supplementary Fig. 6a-d) at
DIV14-15 when compared to DIV10-11. No significant change in ATP levels was detected in neurons expressing Flag vector or WT Mul1 between these two-time points. These data indicate that Mul1 deficiency impairs mitochondrial integrity and thus ATP production at DIV14-15, a time point associated with mitochondrial fragmentation. To further confirm mitochondrial 
Fig. 1 Mul1 restrains neuronal mitophagy under mild stress. a-f Enhanced Parkin recruitment to stressed mitochondria in Mul1-deficient neurons. Cortical neurons at DIV8 were co-transfected with mCherry-Parkin and CFP-Mito together with scr-shRNA, Mul1-shRNA, or co-transfected with mCherry-Parkin and GFP (as baseline), GFP-Mul1, or GFP-Mul1 RRing, followed by incubation at DIV10 with $100 \mathrm{nM}$ AA or DMSO for $3 \mathrm{~h}$ before fixation and imaging. Magnified inserts in boxes show colocalization of Parkin and mitochondria. Note that Mul1 deficiency facilitates more neurons to recruit cytosolic Parkin onto stressed mitochondria, resulting in a higher correlation of Parkin signal on mitochondria (f). To limit variations by unblinded selection, all transfected neurons in low-density culture $\left(0.25\right.$ million per $\left.10 \mathrm{~cm}^{2}\right)$ were selected for analysis. $\mathbf{g}$ Endo-lysosomal targeting of fragmented mitochondria in Mul1deficient neurons in the absence of AA treatment. Low-density cortical neurons were co-transfected using calcium phosphate method at DIV7 with Mul1shRNA, DsRed-Mito, and GFP, followed by immunostaining with LAMP1 at DIV14. GFP signal (pseudo blue) was used to trace transfected neurons. 6-8 transfected neurons were chosen per dish per trial for each condition. $\mathbf{h}, \mathbf{i}$ Enhanced dynamic mitophagy in Mul1-deficient neurons expressing Mul1-shRNA (h) or Mul1 $\Delta$ Ring (i). Cortical neurons at DIV7 were co-transfected with mt-Keima and various constructs as indicated, followed by live imaging at DIV14. $\mathrm{mt}$-Keima is a ratiometric $\mathrm{pH}$-sensitive fluorescent probe that targets to the mitochondrial matrix. A short wavelength ( $458 \mathrm{~nm})$ is predominant for excitation in a neutral environment (mitochondrial signal green), whereas a long wavelength $(561 \mathrm{~nm})$ is predominant in an acidic environment (lysosomal signal red). "Mitophagy Index" was assessed by measuring the ratio of $561 \mathrm{~nm} / 458 \mathrm{~nm}$ in the cell body and normalized to control conditions. Note that Mul1 deficiency enhances dynamic mitophagy. Data were analyzed from the total number of neurons indicated in the bars from three experiments and expressed as mean \pm s.e.m. Unpaired Student's t-test (h), Mann-Whitney test (f, $\mathbf{g})$, or Ordinary one-way ANOVA with Dunnett multiple comparison test $(\mathbf{e}, \mathbf{i}) .\left({ }^{\star \star \star} p<0.001 ;{ }^{\star \star} p<0.01 ;{ }^{\star} p<0.05\right)$. Scale bars: $5 \mu \mathrm{m}(\mathbf{a}-\mathbf{d})$ and $10 \mu \mathrm{m}(\mathbf{g}, \mathbf{h}, \mathbf{i})$. (Also see Supplementary Figs. 1, 2, 3)

dysfunction in bioenergetics, we used the Seahorse XF analyzer to examine the oxygen consumption rate (OCR). Basal and real-time OCR were significantly reduced in Mul1-deficient neurons compared to control (Supplementary Fig. 6e, f).

Our previous study indicated that cortical neurons from parkin $\mathrm{KO}$ mice showed no significant changes in mitochondrial membrane potential and neuronal morphology as compared with wild-type neurons ${ }^{16}$. However, Mull knockdown in parkin $\mathrm{KO}$ neurons resulted in a loss of $\Delta \psi_{\mathrm{m}}$ in the early stage (DIV1011) and dendritic and axonal neurodegeneration. To assess the effect of reduced bioenergetic capacity on neuronal physiology, we examined dendritic arborizations in Mull-deficient neurons by performing Sholl analysis. A significant decrease in dendritic arborization pattern was observed in Mul1-deficient neurons at DIV14-15 compared to control neurons $(p<0.001$, two-way ANOVA test) (Supplementary Fig. 7), indicating that Mull deficiency affects neuronal cell connectivity and survival.

Increased Mfn2 activity mediates Mul1-deficient phenotypes. Deleting Mul1 in Drosophila and suppressing Mul1 in HeLa cells lead to increased Mfn levels ${ }^{16}$. We sought to determine which isoform Mfn1 or Mfn2 plays a prominent role in mediating Mul1-deficient phenotypes. We hypothesize that elevated Mfn1 or Mfn2 in Mul1-deficient neurons accelerates the bi-phasic transition. Expressing either Mfn1 or Mfn2 has a subtle impact on mitochondrial fragmentation in control neurons. However, elevated Mfn2 expression significantly accelerates the transition of mitochondria into fragmentation at DIV10 in Mul1-deficient neurons with Mul1-shRNA ( $p<0.001$, Dunnett's post hoc test) or Mul1 $\Delta$ Ring $(p<0.01)$ compared to Mfn1 expression (Fig. 4a). There was no significant change in percentage of neurons with mitochondrial fragmentation between groups of (1) Mfn1 vs

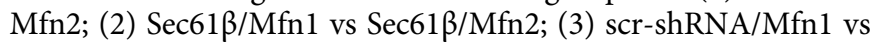
scr-shRNA/Mfn2; and (4) Mul1/Mfn1 vs Mul1/Mfn2. Therefore, these data support that Mfn2, but not Mfn1, accelerates mitochondrial fragmentation in Mull-deficient neurons even at an early stage (DIV10). Given that both Mfn1 and Mfn2 play a similar role in mitochondrial fusion, Mfn2 mediates Mul1deficient phenotypes likely through its non-fusion action.

After validating the specificity of the anti-Mfn2 antibody in cortical neurons by knocking down Mfn2 expression using Mfn2-shRNA-1 or Mfn2-shRNA-2 (Supplementary Fig. 8a-c), we determined whether mitochondria-targeted $M f n 2$ is increased in Mul1-deficient neurons by measuring the Mfn2/cyto $\mathrm{c}$ intensity ratio in dendritic processes. Expressing Mul $1 \Delta$ Ring significantly increases the Mfn $2 /$ cyto $c$ intensity ratio $(p<0.001$, Tukey's post hoc test) while overexpressing Mul1 reduces the ratio $(p<0.001)$ relative to control neurons expressing Flag alone or a non-specific Flag-tubulin (Fig. 4b, c; Supplementary Fig. 8d, e). Second, we sought to establish the causal role of Mfn2 in mediating the Mul1deficient phenotypes by knocking down Mfn2. Cortical neurons were transfected with Mull $\Delta$ Ring at DIV7-8, followed by a second transfection at DIV10-11 with Mfn2-shRNA-1, Mfn2shRNA-2, or control scr-shRNA. Depleting Mfn2 in Mul1deficient neurons rescued mitochondrial fragmentation and size (Supplementary Fig. 8f-h). Third, we performed biochemical analysis showing reduced Mfn2 ubiquitination and increased Mfn2 levels in Mul1-depleted neurons when compared to control neurons (Fig. 4d). In addition, we compared the effect of Mfn2 and a catalytically inactive mutant Mfn2 (K109A) on mitochondrial fragmentation in Mul1-deficient neurons ${ }^{40}$. While expressing Mfn2 triggers an early transition of mitochondria into fragmentation at DIV10, expressing Mfn2 (K109A) reduces mitochondrial fragmentation at DIV14-15 (Fig. 4e, f). To confirm whether elevated Mfn2 mediates Mul1-mutant phenotypes, we expressed Mfn2 alone in control neurons in the absence of stress. Expressing Mfn2, but not Mfn1, triggers mitochondrial fragmentation and Parkin recruitment (Fig. 4g, h). We further measured the dynamic mitophagy using mt-Keima. Neurons with Mfn2 overexpression displayed enhanced lysosomal targeting of mt-Keima over a time course from DIV10-11 to DIV 14-15 $(p<0.001$, unpaired Student's $t$-test) (Fig. $4 \mathrm{i}, \mathrm{j})$. In contrast, expressing Mfn1 has no effect in inducing mitophagy during the same time course. Altogether, these results suggest that elevated Mfn2 activity mediates Mul1-deficient phenotypes.

Elevated Mfn2 impairs ER-Mito contacts. ER-Mito contacts are important to maintain energy metabolism and $\mathrm{Ca}^{2+}$ transfer from the ER to mitochondria $25,26,33,41$. While Mfn1 mainly resides on mitochondria, Mfn2 is sorted to both the mitochondrial OMM and ER, where Mfn2 has been reported to regulate ER-Mito contacts in non-neuronal cells ${ }^{19-22}$. We sought to examine whether Mul1 deficiency alters ER-Mito contacts before mitochondrial fragmentation occurs. First, we measured ER-Mito contact sites in neurons by applying 3D diffraction-unlimited stimulated emission depletion (3D-STED) nanoscopy that can reach a lateral resolution of 30-70 $\mathrm{nm}$. ER structures were labeled by ER membrane marker GFP-Sec61 $\beta$ and mitochondria were stained with an antibody against Tom20. ER-Mito contact sites were measured at DIV10-11 by calculating Mander's colocalization coefficient. Cortical neurons expressing Mul1 $\Delta$ Ring display reduced ER-Mito contacts $(p<0.05$ or $p<0.01$, one-way ANOVA) in soma-dendritic or distal dendritic regions, respectively, while expressing Mul1 enhances ER-Mito contacts relative 
a
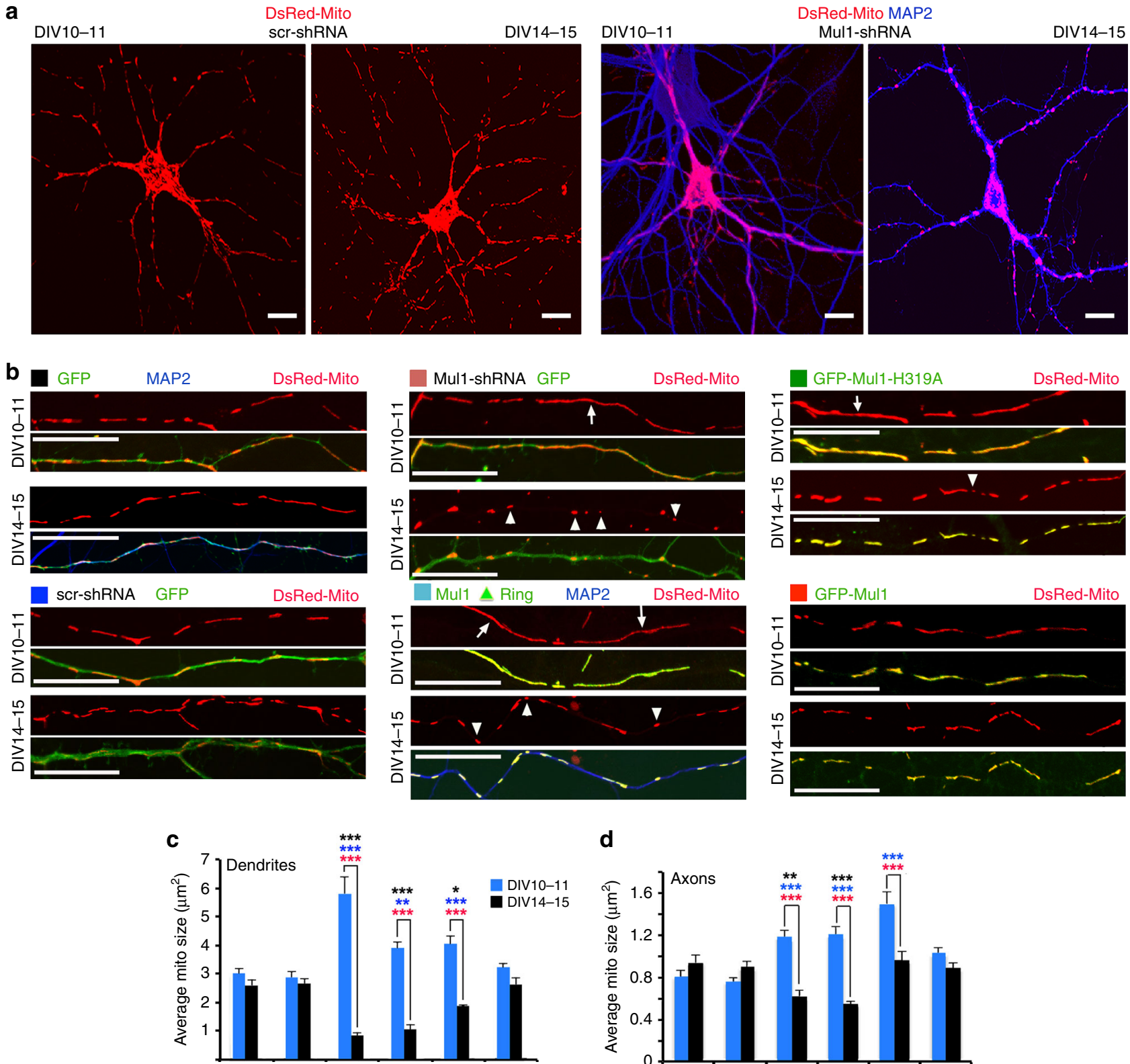

d
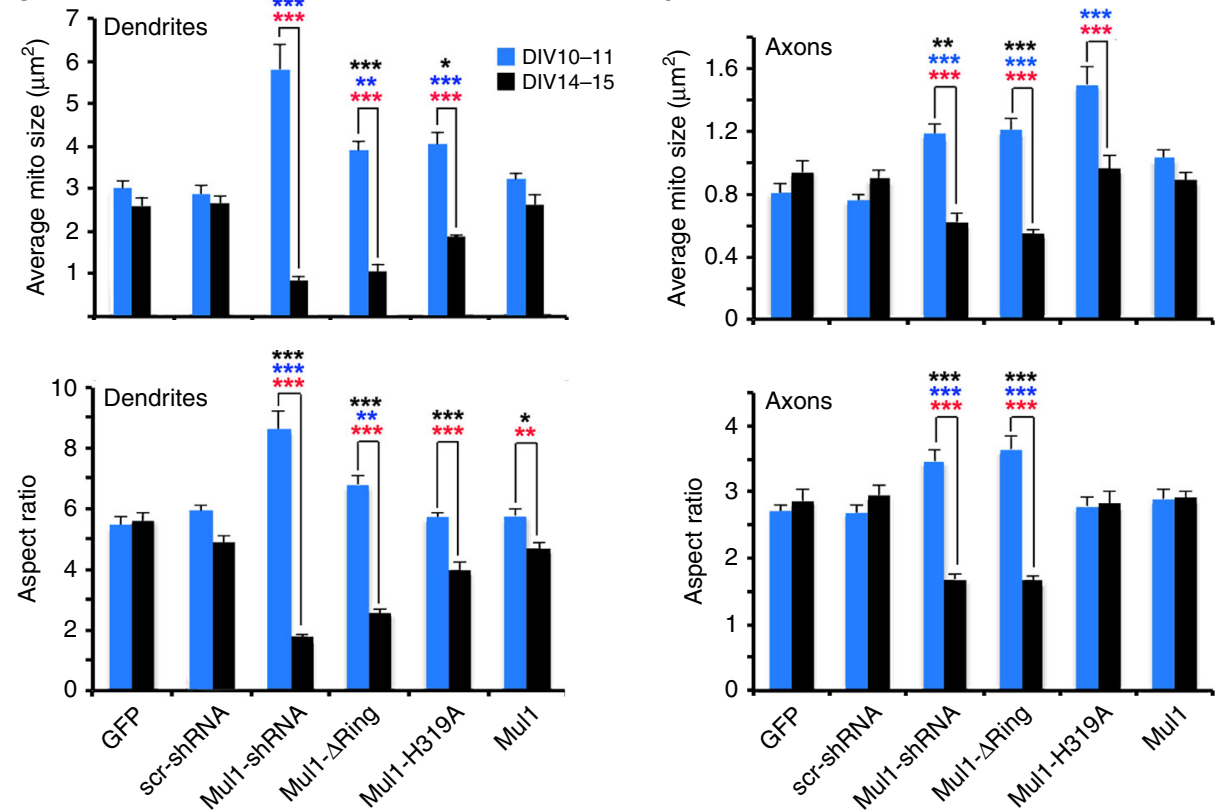

to control $(p<0.05)$ (Fig. 5a, b, Supplementary Videos 1-3). Since expressing Mull does not result in a dramatic change in mitochondrial morphology (Fig. 2b-d), Mull likely has a primary role in regulating ER-Mito contact under normal physiology.

We next confirmed the super-resolution imaging with immunotransmission electron microscopy (iTEM) analysis. First, the ER targeting of GFP-Sec61 $\beta$ was examined by immunostaining

cortical neurons with an anti-GFP antibody and a 10-nm goldlabeled secondary antibody. Gold-labeled GFP-Sec61 $\beta$ is selectively scattered along the surface of ER membranes (Fig. 5c). The ER-Mito contact area were measured if the distance between the two organelles was $<12 \mathrm{~nm}$, as previously reported ${ }^{19-21}$. While overexpressing Mul1 enhances ER-Mito contact $(p<0.001$, Dunnett's post hoc test) relative to control neurons, expressing 
Fig. 2 Mul1-deficient neurons display a biphasic change in mitochondrial morphology. a Transient mitochondrial hyperfusion followed by fragmentation in Mul1-depleted neurons. Cortical neurons were co-transfected at DIV7-8 with DsRed-Mito and control scr-shRNA or Mul1-shRNA, followed by immunostaining with MAP2 at DIV10-11 or DIV14-15. Note that neurons with Mul1 depletion display a transition from mitochondrial hyperfusion at DIV1011 to fragmentation at DIV14-15. b-d Mitochondrial size and aspect ratio in dendrites and axons, across different conditions for phase I (DIV10-11) and phase II (DIV14-15). Cortical neurons at DIV7-8 were co-transfected with DsRed-Mito and GFP + scr-shRNA or GFP + Mul1-shRNA, or GFP-Mul1, Mul14Ring, or Mul1-H319A mutants, followed by imaging at DIV10-11 and DIV14-15. Note that the biphasic transition was consistently observed in Mul1deficient neurons: hyperfused tubular mitochondria (arrows) at DIV10-11 and fragmented ones in dendrites (arrowheads) at DIV14-15 were observed in neurons transfected with Mul1-shRNA, Mul1 $\Delta$ Ring, or Mul1-H319A. Data were analyzed from more than three experiments and the total number of neurons for dendritic mitochondria groups (c): GFP (55), scr-shRNA (58), Mul1-shRNA (36), Mul1 $\Delta$ Ring (58), Mul1-H319A (41), and Mul1 (62); and for axonal mitochondria groups (d): GFP (23), scr-shRNA (34), Mul1-shRNA (44), Mul1 $\Delta$ Ring (20), Mul1-H319A (20), and Mul1 (26). Data were expressed as mean \pm s.e.m. Two-way ANOVA with Sidak's multiple comparison test (c, d) was used for comparing two-time-phase groups (DIV10-11 vs DIV14-15) within each transfection condition (red ${ }^{\star \star \star} p<0.001$; red ${ }^{\star \star} p<0.01$ ). Two-way ANOVA with Holm-Sidak's multiple comparison test was used for comparing GFP control with other transfection conditions across the phase I group (DIV10-11: blue bars and blue ${ }^{\star \star *} p<0.001$; blue ${ }^{\star} p<0.05$ ), or across the phase II group (DIV14-15: black bars and black ${ }^{\star \star \star} p<0.001$; black ${ }^{\star \star} p<0.01$ ). Scale bars: $10 \mu \mathrm{m}$ (a), $5 \mu \mathrm{m}$ (b). (Also see Supplementary Fig. 4, 5a-d)

Mul1 $\Delta$ Ring or knocking down Mul1 in neurons significantly reduces ER-Mito contact $(p=0.01, p<0.001$, respectively, Mann-Whitney $U$-test) (Fig. $5 \mathrm{~d}$-g). In addition, overexpressing Mfn2, but not Mfn1, also inhibits ER-Mito contact $(p<0.001$, Dunnett's post hoc test). Altogether, our super-resolution imaging and ultrastructural analysis consistently indicate that Mull protects neuronal mitochondria integrity by maintaining ERMito contacts; impaired ER-Mito contacts in Mull-deficient neurons is mediated through elevated Mfn2 expression.

ER-Mito contacts play an important role in $\mathrm{Ca}^{2+}$ transfer from ER to mitochondria that is essential for bioenergetics $25,26,33,41$. Thus, temporal measurement of $\mathrm{Ca}^{2+}$ uptake by mitochondria following physiological $\mathrm{Ca}^{2+}$ release from ER is an important functional readout of ER-Mito coupling ${ }^{42,43}$. We applied a genetically encoded FRET-based ratiometric $\mathrm{Ca}^{2+}$ probe $(4 \mathrm{mtD} 3 \mathrm{cpv})$ that specifically targets the mitochondrial matrix ${ }^{44,45}$. The emission spectra $(525 \mathrm{~nm} / 458 \mathrm{~nm})$ were obtained by exciting $405 \mathrm{~nm}$ before and upon stimulation with $10 \mu \mathrm{M}$ histamine. Mul1deficient neurons display a reduced mitochondrial $\mathrm{Ca}^{2+}$ peak $(p<$ 0.001 , unpaired Student's $t$-test) following histamine-induced release from the ER (Fig. 6a, b). Alternatively, we constructed a non-tagged Mull-shRNA for calcium imaging experiment. Depleting Mull in neurons reduces mitochondrial $\mathrm{Ca}^{2+}$ uptake from the ER (Supplementary Fig. 9). We further examined the mitochondrial $\mathrm{Ca}^{2+}$ load in both dendritic and axonal compartments at DIV10-11. The pseudo colors cyan (458 nm) and green $(525 \mathrm{~nm})$ were imaged when excited with $405 \mathrm{~nm}$. The mitochondrial $\mathrm{Ca}^{2+}$ load was expressed as a ratio $(525 \mathrm{~nm} / 458 \mathrm{~nm})$. Expressing Mul $\Delta$ Ring significantly reduces the ratio in both dendritic and axonal regions ( $p<0.01$, Dunnett's post hoc test) while expressing Mul1 enhances the ratio $(p<0.05)$, indicating an altered mitochondrial $\mathrm{Ca}^{2+}$ load (Fig. 6c, d). Mul1-deficient neurons, where hyperfused mitochondria are readily detected at DIV10-11, display reduced mitochondrial $\mathrm{Ca}^{2+}$ uptake from the $\mathrm{ER}$, reflecting an impaired ER-Mito interplay.

Impaired ER-Mito contact induces mitochondrial fragmentation. By immunostaining cortical neurons with an anti-Drp1 antibody at DIV14-15, we determined whether Drp1 is recruited to OMM after ER-Mito contacts are impaired. Drp1 puncta mainly co-localize with fragmented mitochondria, suggesting Drp1 recruitment for fission. Depleting Mul1 significantly enhances the density of Drp1 puncta along dendritic mitochondria (Fig. 7a-c). To confirm the role of Drp1 in the second phasic mitochondrial fragmentation, we performed mitochondrial fractionation of WT and Mul1-depleted neurons at DIV12-13. An increased recruitment of total Drp1 $(p<0.005$, unpaired Student's $t$-test) into the mitochondrial fraction was detected in Mul1depleted neurons when compared to WT neurons (Fig. 7d). The drp1 gene contains several alternative exons and produces multiple isoforms through RNA splicing 46 , in which the shorter isoform named isoform 12 encoding 587 residues contains all functional domains. This shorter isoform $(\sim 58 \mathrm{kDa})$ is robustly increased in the mitochondrial fraction in Mull-deficient neurons. Interestingly, recruitment of this isoform into mitochondria is largely suppressed in control neurons (Fig. 7d). In addition to the long Drp1 isoform, it is likely that this shorter Drp1 isoform plays a role in the second phasic mitochondrial fragmentation. Expressing Drp1 loss-of-function mutant Drp1K38A in Mul1deficient neurons locks mitochondria in the hyperfusion status at DIV14-15, while expressing Mul1-shRNA or Mul1 $\Delta$ Ring alone display robust mitochondrial fragmentation at the same stages (Fig. 7e-g). Thus, our study supports that the second phasic fragmentation is a Drp1-dependent process. Next, we asked how Drp1 is activated to trigger mitochondrial fragmentation in Mul1deficient neurons. Drp1 recruitment and fission activity is primarily regulated by post-translational modifications. Increased cytoplasmic $\mathrm{Ca}^{2+}$ load activates calcineurin phosphatase which dephosphorylates Drp1 at serine 637 and thus activates Drp $1^{47}$. Our immunoblot analysis confirmed a suppressed phosphorylation status of Drp1 in Mul1-depleted neurons (Supplementary Fig. 8i). These findings suggest that defective ER-Mito coupling builds cytoplasmic $\mathrm{Ca}^{2+}$ load that subsequently activates Drp1. To test this, we expressed a cytoplasm-targeted FRET-based ratiometric $\mathrm{Ca}^{2+}$ probe $(\mathrm{D} 3 \mathrm{cpv})^{44,45}$ in cortical neurons at DIV78 , followed by live imaging at DIV12-13 just before mitochondrial fragmentation occurs. Mul1-deficient neurons display an increased cytoplasmic $\mathrm{Ca}^{2+}$ peak $(p=0.01$, unpaired Student's $t$ test) following histamine-induced release from the ER (Fig. 8a, b).

We further examined whether calcineurin activity is associated with mitochondrial fragmentation by applying two inhibitors of calcineurin, cyclosporine A $(\mathrm{CsA})^{48}$ and FK506 ${ }^{49}$. Cortical neurons, co-transfected with DsRed-Mito and Mul1shRNA or scr-shRNA at DIV7-8, were treated with CsA $(0.5 \mu \mathrm{M})$ or FK506 $(0.6 \mu \mathrm{M})$ for $48 \mathrm{~h}$ starting at DIV12. Strikingly, fragmentation was largely abolished in Mul1-depleted neurons following either treatment of CsA or FK506 (Fig. 8c, d). Blocking calcineurin activity locks mitochondria in the hyperfusion status at DIV14. Thus, our study provides new mechanistic insights as to how impaired ER-Mito interplay triggers mitochondrial fragmentation through elevated cytoplasmic $\mathrm{Ca}^{2+}$ load and calcineurin activity.

Enhancing ER-Mito coupling suppresses neuronal mitophagy. ER-Mito contacts are tethered by proteins between the two organelles, including mitochondrial protein PTPIP51 and ER protein $\mathrm{VAPB}^{23}$. To support our conclusion, we sought to enhance the ER-Mito coupling in Mull-deficient cortical neurons by co-expressing HA-tagged PTPIP51. First, we 
a
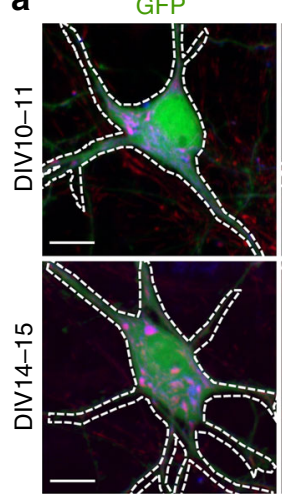

b Scr-shRNA GFP
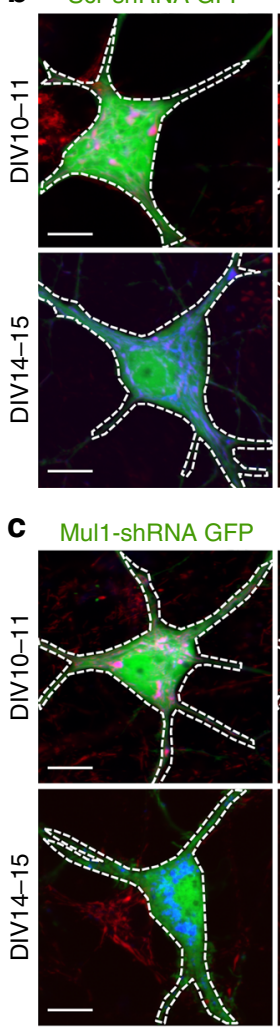

d MUL1 A RING

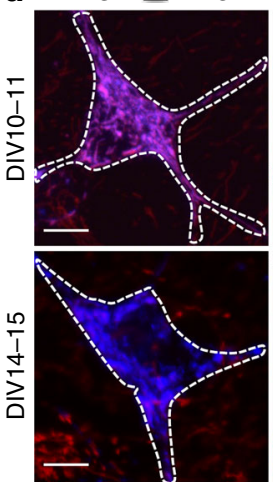

TMRE
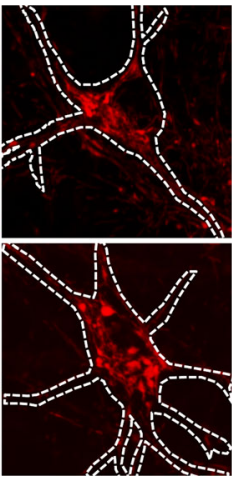

TMRE

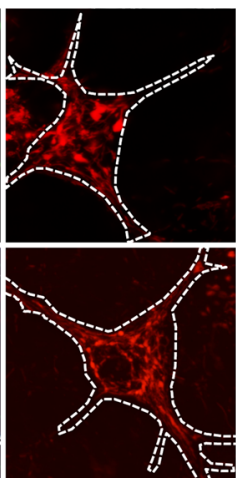

TMRE

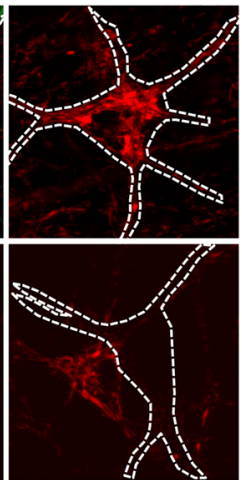

TMRE

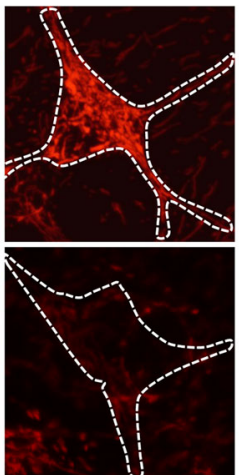

CFP-Mito
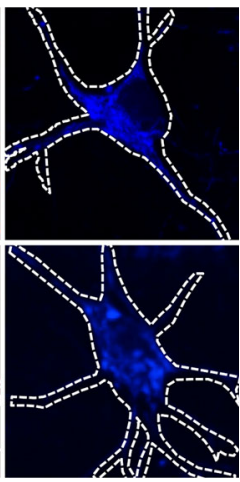

CFP-Mito

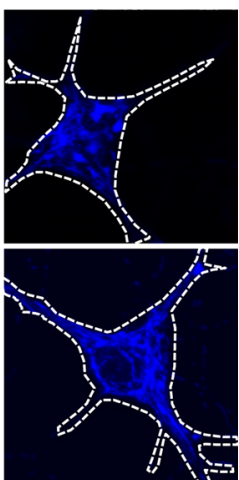

CFP-Mito

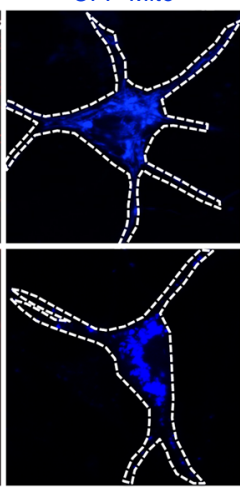

CFP-Mito
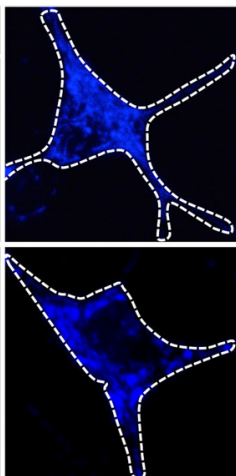

e GFP-Mul1-H319A
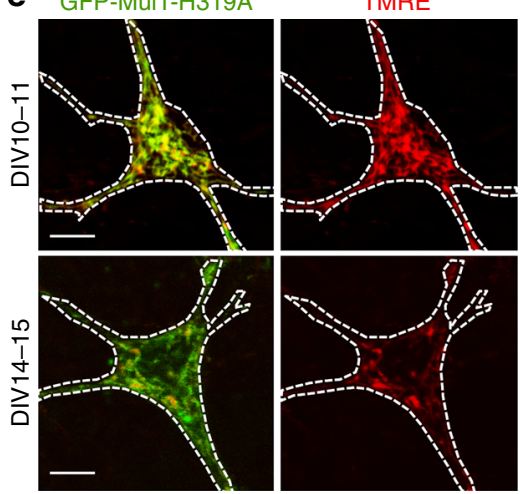

f GFP-Mul1
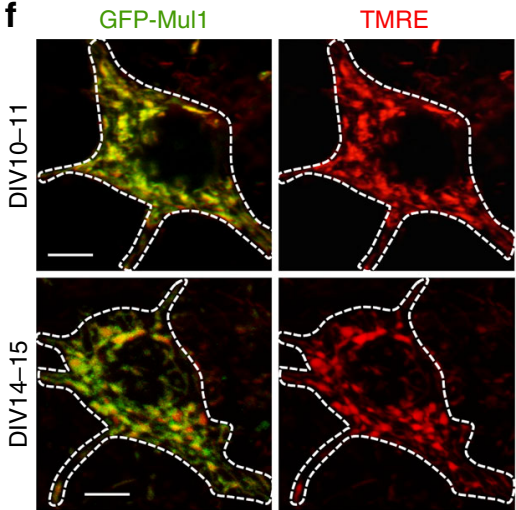

g
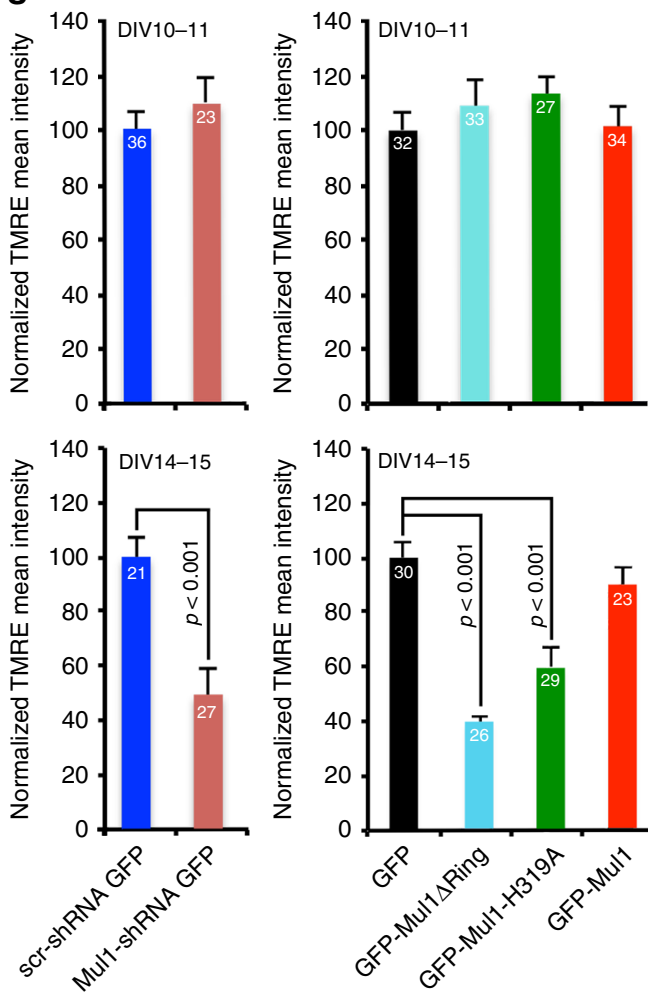

confirmed mitochondria-targeting of PTPIP51 in neurons (Fig. 9a). Second, by tracing the average fluorescence ratio (Fig. 9b) and measuring mitochondrial $\mathrm{Ca}^{2+}$ peak ratio $(525 \mathrm{~nm} / 458 \mathrm{~nm})$ (Fig. 9c) following stimulation with $10 \mu \mathrm{M}$ histamine, we found a partial rescue of mitochondrial $\mathrm{Ca}^{2+}$ load by PTPIP51 expression in Mul1-deficient neurons. Third, expressing PTPIP51 rescues mitochondrial fragmentation (Fig. 9d-e) and significantly suppresses Parkin translocation to mitochondria (Fig. 9g). Thus, our study consistently supports that enhancing ER-Mito contacts rescues Mull-deficient 
Fig. 3 Mul1 deficiency reduces mitochondrial $\Delta \psi_{\mathrm{m}}$ in mature neurons. Cortical neurons were co-transfected at DIV7-8 with GFP and CFP-Mito (a), GFP, CFP-Mito and scr-shRNA (b), GFP, CFP-Mito and Mul1-shRNA (c), CFP-Mito and Flag-Mul1 2 Ring (d), GFP-Mul1-H319A (e), or GFP-Mul1 (f).

Mitochondria were labeled by CFP-Mito (a-d: blue channel) or by mitochondria-targeted GFP-Mul1-H319A (e) or GFP-Mul1 (f) (green channel). Live imaging was performed at both DIV10-11 and DIV14-15 following loading of the $\Delta \psi_{\mathrm{m}}$-dependent fluorescent dye TMRE (50 nm) for $20 \mathrm{~min}$. Note that while neurons expressing GFP (a), scr-shRNA (b), or GFP-Mul1 (f) display TMRE-labeled polarized mitochondria at both DIV10-11 and DIV14-15, Mul1-deficient neurons (c-e) results in loss of TMRE staining of mitochondria at DIV14-15 (g), indicating mitochondrial depolarization. The edges of transfected cell bodies and proximal processes are marked with white dashed lines. TMRE mean intensity was measured and normalized to control neurons transfected with GFP at both time points. The data were analyzed from the total number of neurons shown in the bars from three experiments and expressed as mean \pm s.e.m. Unpaired Student's $t$-test was used for comparing two groups and Ordinary one-way ANOVA with Dunnett multiple comparison test for multiple comparisons. Scale Bars: $10 \mu \mathrm{m}$. (Also see Supplementary Figs. 5e, f, and 6)

a

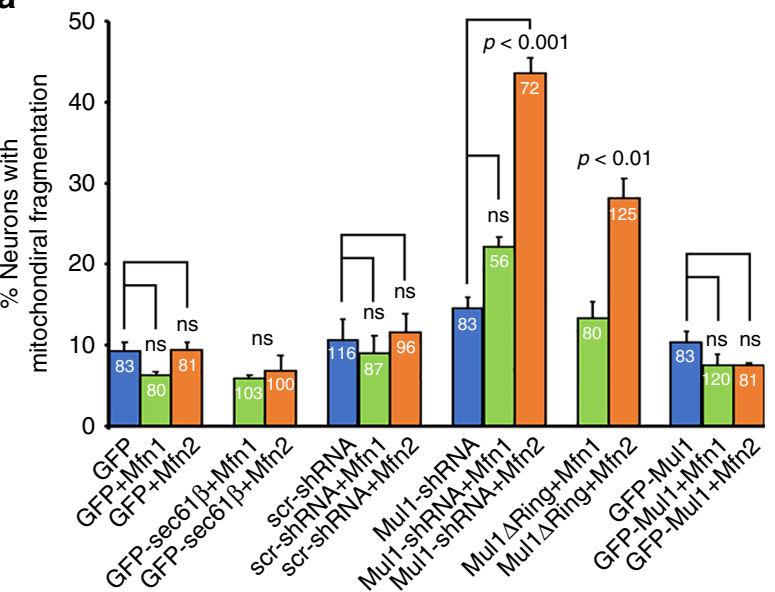

d

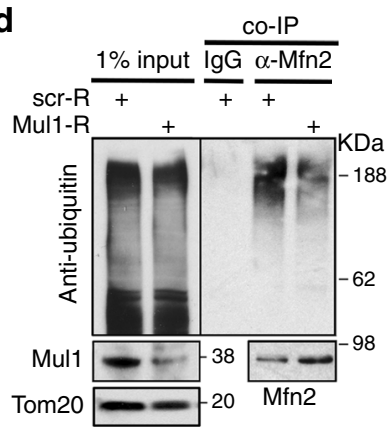

b
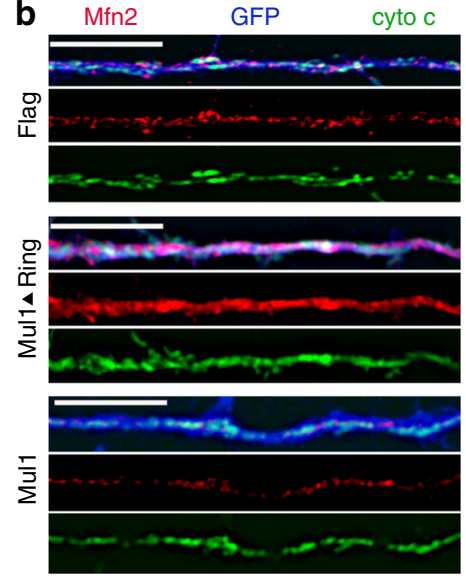

f

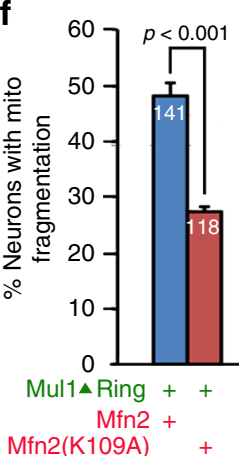

C

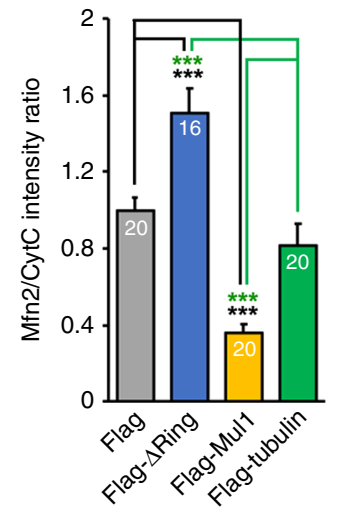

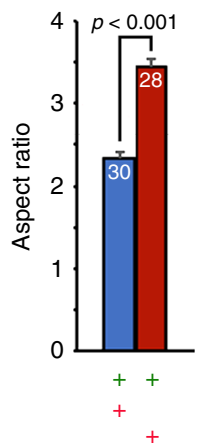

g

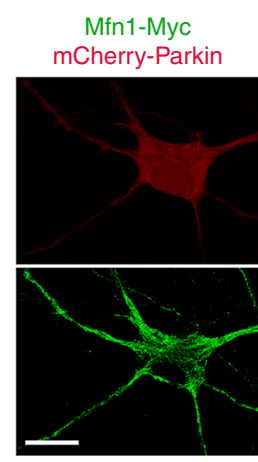

Mfn2-Myc mCherry-Parkin

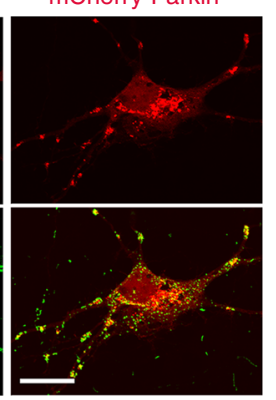

h

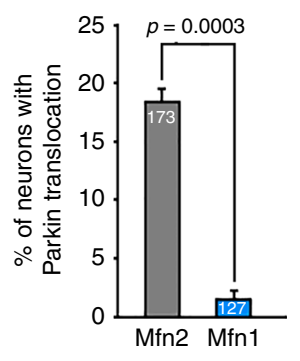

j

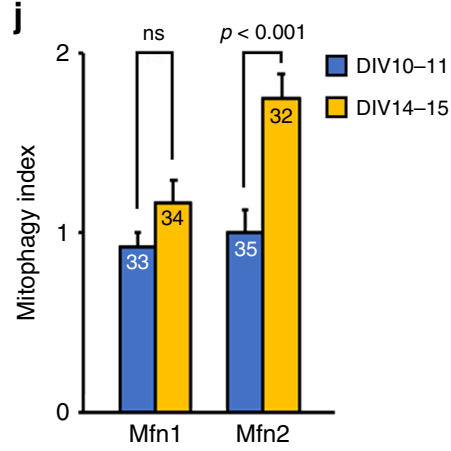

i
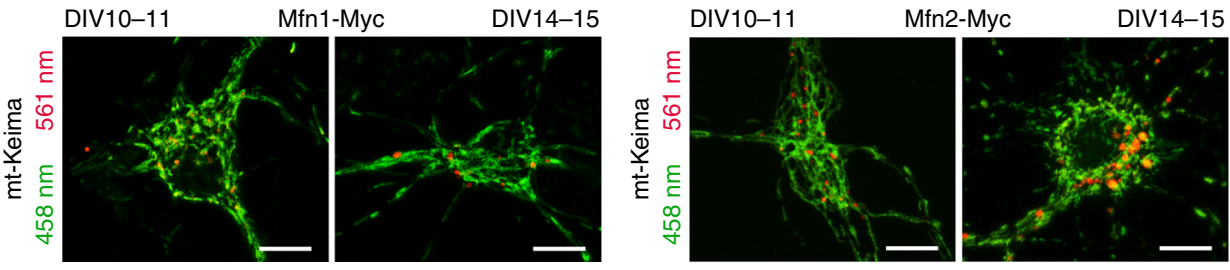
Fig. 4 Elevated Mfn2 activity mediates Mul1-deficient phenotypes. a Accelerated mitochondrial fragmentation at DIV10 following elevated Mfn2 expression in Mul1-deficient neurons. Cortical neurons at DIV7 were co-transfected with various combinations of expression vectors as indicated, followed by imaging mitochondria at DIV10. b, c Significant changes in Mfn2 levels in dendrites of Mul1-deficient neurons. Cortical neurons at DIV7 were cotransfected with various combinations of expression vectors as indicated, followed by co-immunostaining of Mfn2 and cytochrome c (cyto c) at DIV10. GFP signals (pseudo blue) were used to trace transfected neurons. Relative Mfn2 levels were determined by measuring the intensity ratio of Mfn2/cyto $c$ and normalized to control neurons expressing Flag. Note that expressing Flag-Mul1 $\Delta$ Ring increases normalized Mfn2/cyto $c$ intensity ratio while expressing Mul1 reduces the ratio relative to control neurons expressing Flag alone $\left(p<0.001\right.$, black $\left.{ }^{\star \star \star}\right)$ or Flag-tubulin $\left(p<0.001\right.$, green $\left.{ }^{\star \star \star}\right)$. $\mathbf{d}$ Reduced Mfn2 ubiquitination and increased Mfn2 levels in Mul1-depleted cortical neurons. Neurons were transduced with Mul1-shRNA or scr-shRNA, followed by sequential blotting on the same blots with antibodies as indicated. e, f Reduced mitochondrial fragmentation by expressing Mfn2(K109A). Cortical neurons were co-transfected at DIV7-8 with Flag-Mul14Ring and Mfn2-Myc or Mfn2(K109A)-Myc, followed by co-immunostaining at DIV10-11. The percentage of neurons with mitochondrial fragmentation was determined from the total number of neurons indicated in the bars. Average mitochondrial size and aspect ratio were analyzed from a total of 8423 and 8354 mitochondria, respectively. $\mathbf{g}$, $\mathbf{h}$ Enhanced Parkin translocation to mitochondria upon overexpression of Mfn2, but not Mfn1, in the absence of AA. Cortical neurons were co-transfected at DIV7-8 with mCherry-Parkin and Myc-tagged Mfn2 or Mfn1, followed by immunostaining Myc-tag at DIV14-15. i, j Enhanced mitophagy process upon overexpression of Mfn2, but not Mfn1, at DIV14-15. Data were analyzed from the total number of neurons indicated in the bars and expressed as mean \pm s.e.m. Unpaired Student's $t$-test was used for comparing two groups (f, $\mathbf{h}$, j) and Ordinary one-way ANOVA with Dunnett's post hoc test (a) or Tukey's multiple comparison test (c) for multiple comparisons. Scale bars: $10 \mu$ m. (Also see Supplementary Fig. 8)

mitochondrial phenotypes and thus suppresses Parkinmediated mitophagy in neurons.

\section{Discussion}

In the current study, we reveal Mull as an early checkpoint to protect neuronal mitochondria from rapid degradation under mild stress conditions, thus maintaining energy supply and ensuring cell survival. Mul1 deficiency leads to increased Mfn2 activity that plays two parallel roles: (1) inducing the first phasic mitochondrial hyperfusion; and (2) impairing ER-Mito interplay, the latter disturbs mitochondrial bioenergetics and $\mathrm{Ca}^{2+}$ homeostasis. Elevated cytoplasmic $\mathrm{Ca}^{2+}$ activates Drp1 through calcineurin, triggering the second phasic mitochondrial fragmentation and Parkin-mediated mitophagy. Consistently, stabilizing ERMito contacts by expressing anchoring protein PTPIP51 partially rescues Mul1-deficient phenotypes. Thus, impaired ER-Mito contacts and disturbed $\mathrm{Ca}^{2+}$ homeostasis link to mitochondrial fragmentation and mitophagy (Fig. 10). Our study provides new mechanistic insights into (1) why mitophagy in mature neurons is observed only in a small proportion of mitochondria following depolarization $^{5,7}$ and occurs much more slowly than in nonneuronal cells ${ }^{5,6}$; and (2) why Parkin or PINK1 knock-out mice showed only subtle changes in mitochondrial morphology and neuronal degeneration $8,9,50,51$. This Mul1-Mfn2 pathway is particularly relevant to neurodegenerative diseases associated with chronic mitochondrial dysfunction and altered ER-Mito interplay $1,3,23,52$

Maintaining a healthy pool of mitochondria in distal axons and dendrites is more protective than rapidly eliminating them through mitophagy. Our previous studies using mature cortical neurons demonstrated delayed Parkin-mediated mitophagy ${ }^{5,6}$, arguing for unique mechanisms that maintain and/or recover mitochondrial integrity before mitophagy activation. It was reported that Mull and Parkin mediate two parallel pathways of mitochondrial quality control in HeLa cells following acute depolarization using high-dose AA $(40-80 \mu \mathrm{g} / \mathrm{ml} \text { or } 73-146 \mu \mathrm{M})^{16}$. Our study aimed to address whether Parkin-mediated mitophagy is the second resort for mitochondrial quality control after early protection mechanisms have failed. To address this issue, we instead applied mild stress conditions (100 nM AA), a $~ 400-800 \times$ lower dose of AA than widely used in the literature, in mature cortical neurons. We reasoned that this mild stress is more relevant to chronic mitochondrial dysfunction associated with aging-linked neurodegenerative diseases ${ }^{6}$. We established a Mull-deficient neuronal system, where Parkin recruitment to fragmented mitochondria is robustly increased in response to mild mitochondrial stress. In contrast, overexpressing Mul1 suppresses Parkin translocation. Mull loss of function triggers a biphasic transition from mitochondrial hyperfusion at DIV10-11 to fragmentation at DIV1415. Those fragmented mitochondria are associated with loss of membrane potential and ATP production and are subjected to mitophagic clearance. Since overexpressed Mul1 $\Delta$ Ring targets to mitochondria, we speculate that Mull $\Delta$ Ring competes with relatively low levels of endogenous Mull for its interaction or ubiquitination of Mfn2, thus interfering with Mito-ER contact and inducing mitochondrial fragmentation leading to mitophagy. Given that Parkin-mediated mitophagy is rapidly activated in HeLa cells upon mitochondrial depolarization, our study highlights a unique checkpoint for neuronal mitochondrial quality control. After this pathway has failed, Parkin is recruited to fragmentated mitochondria to initiate mitophagy. This is consistent as the loss of both Mull and parkin aggravates mitochondrial damage and induces neuronal degeneration ${ }^{16}$.

As a mitochondrial E3 ligase, Mul1 regulates Mfn levels in flies ${ }^{16}$ and both Mfn1 and Mfn2 levels in Hela cells ${ }^{53}$. However, it is not known which Mfn isoform is involved in Mull mutant phenotypes in neurons. Our current study provides multiple lines of analysis showing that Mfn2 but not Mfn1 mediates Mul1deficient phenotypes. First, expressing Mfn2, but not Mfn1, in Mul1-deficient neurons accelerated mitochondrial fragmentation at the early stage (DIV10). Second, neurons expressing Mfn2 but not Mfn1 displayed a significant increase in Parkin translocation to stressed mitochondria at late stage (DIV14-15). Third, neurons expressing Mfn2 but not Mfn1 displayed enhanced mitophagy over a time course from DIV10-11 to DIV14-15. Fourth, mitochondria-targeted Mfn2 was increased in Mul1-deficient neurons. Fifth, Mul1-depleted cortical neurons displayed reduced Mfn2 ubiquitination and increased Mfn2 levels. In addition, expressing Mfn2 but not catalytically inactive mutant Mfn2 (K109A) induced an early transition of mitochondria into fragmentation at DIV10. Altogether, these results consistently support our notion that elevated Mfn2 activity is sufficient to mediate Mul1-deficient phenotypes in neurons.

Our study also reveals unique mitochondrial phenotypes in Mul1 mutant neurons: transient hyperfusion as an early response to maintain bioenergetic capacity. Prolonged stress impairs ERMito interplay and disturbs mitochondrial functions and $\mathrm{Ca}^{2+}$ homeostasis. Elevated cytoplasmic $\mathrm{Ca}^{2+}$ load activates Drp1 through calcineurin, thus triggering mitochondrial fragmentation. We provided six lines of evidence to support this mechanistic model: (1) mitochondrial association of Drp1 is increased at DIV13, a time point just before fragmentation occurs; (2) 
a
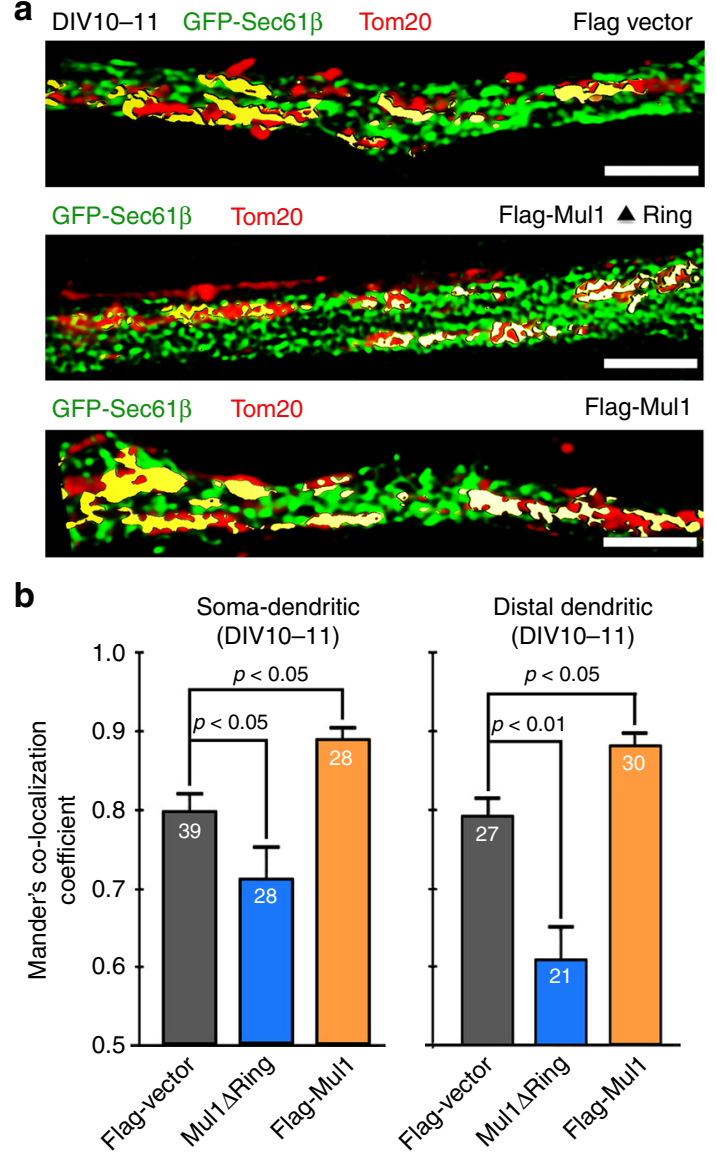

C Cortical neuron (DIV10-11)
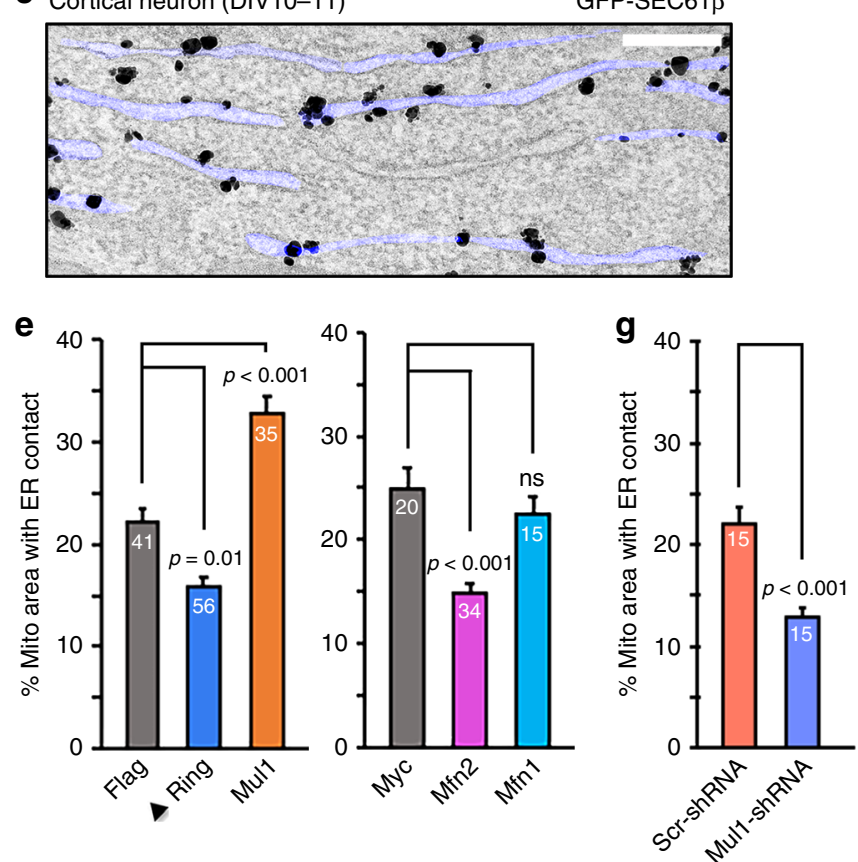

mitochondria are locked at the hyperfusion state when expressing catalytically inactive mutant Drp1K38A; (3) cytoplasmic $\mathrm{Ca}^{2+}$ load is elevated in Mul1-deficient neurons; (4) Drp1 is activated through the $\mathrm{Ca}^{2+} /$ calcineurin when ER-Mito contacts are impaired; (5) expressing ER-Mito anchoring protein PTPIP51 rescues mitochondrial fragmentation. We further confirmed this model by applying two blockers of calcineurin activity, which
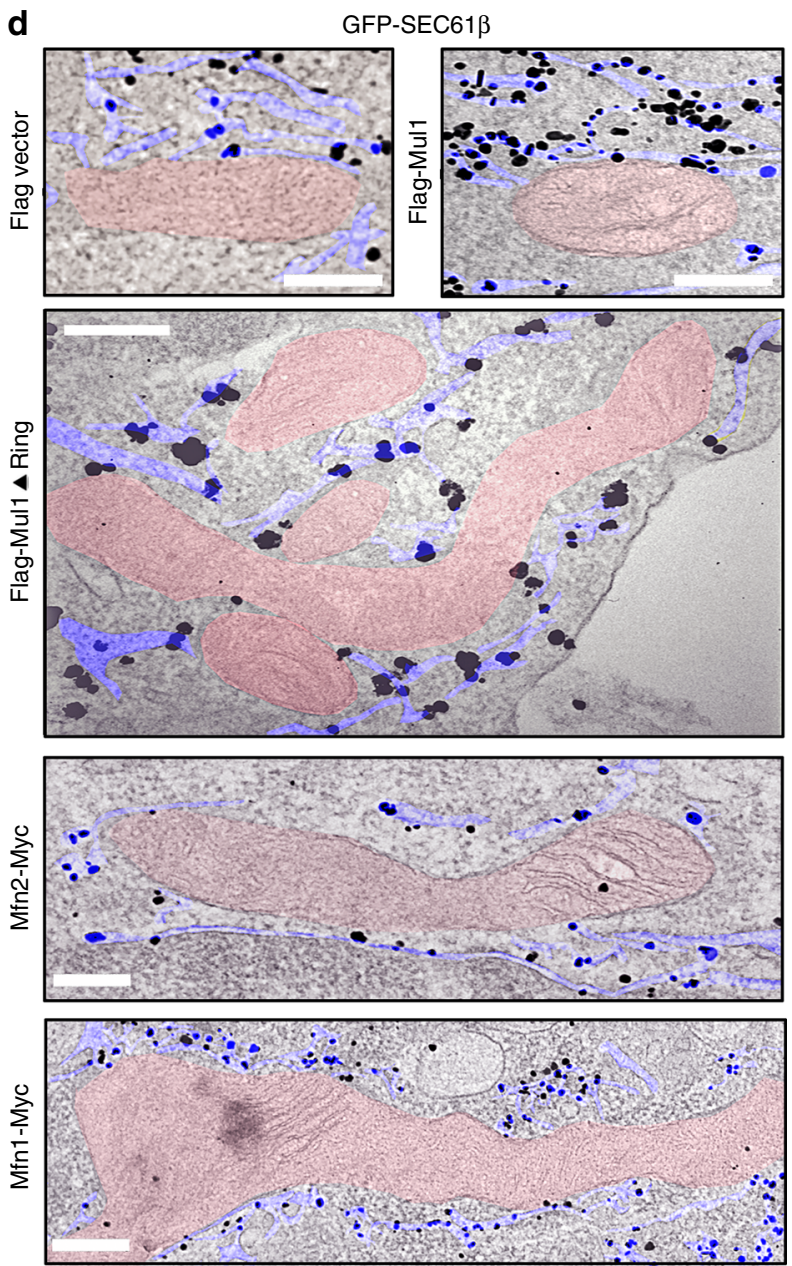

f mcherry-SEC61 $\beta$
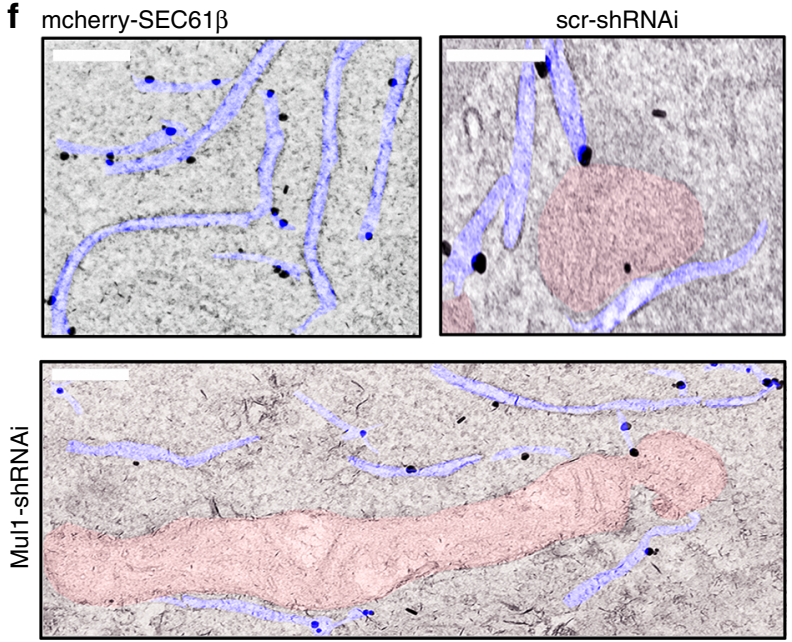

arrest mitochondria in the hyperfusion state in Mul1-depleted neurons. Thus, our study reveals a new mechanistic pathway that links impaired ER-Mito contacts to Drp1-dependent mitochondrial fragmentation, a process necessary for Parkin-mediated mitophagy.

Transient mitochondrial hyperfusion was proposed as an adaptive response to maintain bioenergetic status under various 
Fig. 5 Mul1 deficiency reduces ER-Mito contacts. a, b Representative STED images and quantitative analysis showing reduced ER-Mito contacts in dendrites of Mul1-deficient neurons. Cortical neurons were co-transfected at DIV7 with the ER marker GFP-Sec61 $\beta$ and Flag, Flag-Mul1 $\Delta$ Ring, or Flag-Mul1. Neurons were fixed at DIV11 and immunostained for Tom20. Super-resolution images were captured using dual-color 3D-STED nanoscopy. The relative colocalization between the ER and mitochondria was measured in both somato-dendritic and distal dendritic regions with a plug-in from Volocity Software and expressed as Mander's colocalization coefficient. The yellow color represents overlap between the ER and mitochondria. Note that expressing Mul1 $\Delta$ Ring significantly reduces ER-Mito contacts while expressing Mul1 enhances the contacts relative to Flag baseline control. c-g Representative iTEM graphs and quantitative analysis showing reduced ER-Mito contacts in Mul1-deficient neurons. Neurons at DIV7-8 were co-transfected with GFP-Sec61 $\beta$ and Flag, Flag-Mul1, Flag-Mul1 Ring, Myc, Mfn1-Myc or Mfn2-Myc, followed by immunostaining with an anti-GFP antibody (c, d); or co-transfected with mcherry-Sec61 $1 \mathrm{~b}$ and scr-shRNA or Mul1-shRNA followed by immunostaining with an anti-mCherry antibody (f) and stained with a 10-nm gold-labeled secondary antibody at DIV10-11. A representative iTEM image shows specific gold-labeling of ER membranes with an antibody against GFP (c) or mCherry (f). The ER tubular structures were colored with light blue while mitochondria were colored with light pink. The percentage of mitochondrial surface area contacting with ER were quantified by measuring the distance within a range of $<12 \mathrm{~nm}$ between ER and mitochondrial outer membrane. Note that expressing Flag-Mul1 $\Delta$ Ring, Myc-Mfn2, or Mul1-shRNA significantly reduces ER-Mito contacts relative to control. All data were analyzed from the total number of neurons $(\mathbf{b})$ or mitochondria $(\mathbf{e}, \mathbf{f})$ shown in the bars in three experiments and expressed as mean \pm s.e.m. Ordinary one-way ANOVA with Dunnett's post hoc test (b, e) and Mann-Whitney U-test (g). Scale Bars: $1 \mu \mathrm{m}$ (a) or $100 \mathrm{~nm}(\mathbf{c}, \mathbf{d}, \mathbf{f})$

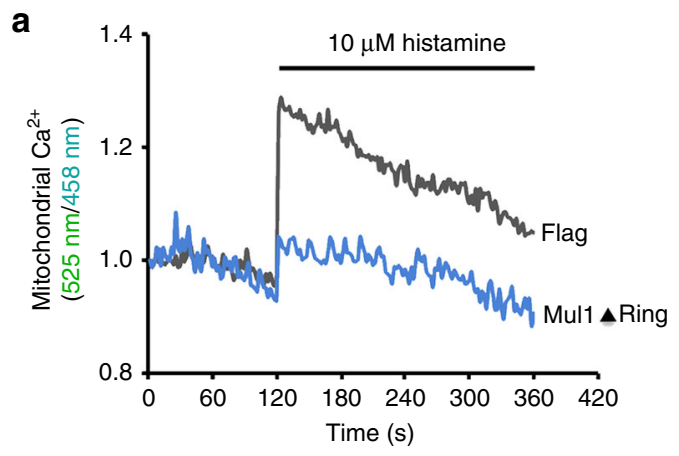

C Dendrites (DIV10-11)
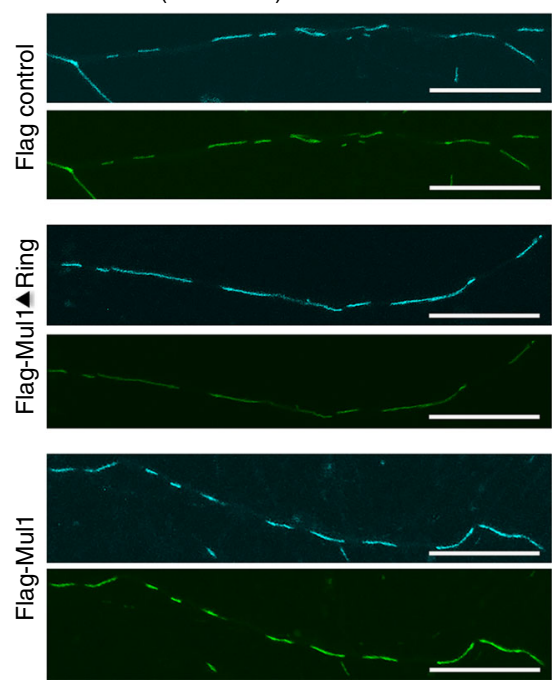

b

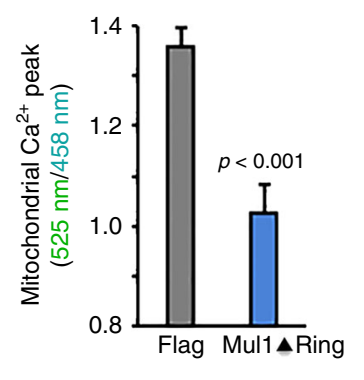

Axons (DIV10-11)
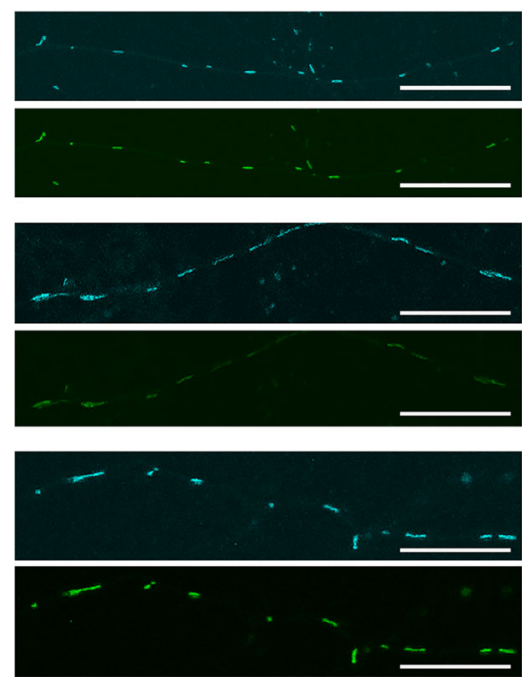
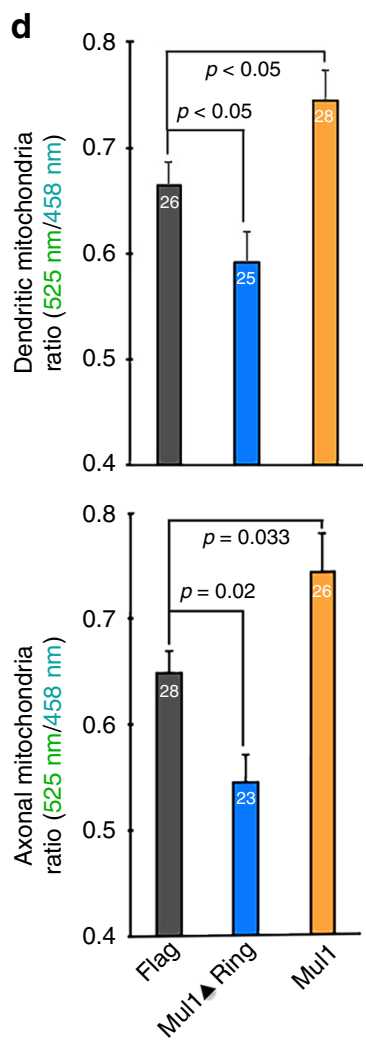

Fig. 6 Mul1 deficiency reduces mitochondrial $\mathrm{Ca}^{2+}$ uptake from the ER. a, b Representative traces and quantitative analysis of the average maximal Ca ${ }^{2+}$ peak showing impaired mitochondrial $\mathrm{Ca}^{2+}$ uptake from the ER in Mul1-deficient neurons. Cortical neurons were co-transfected at DIV7-8 with pcDNA$4 \mathrm{mtD} 3 \mathrm{cpv}$ and Flag or Flag-Mul1 $\Delta$ Ring, followed by live imaging at DIV10-11. The emission spectra of the ratiometric probe $(525 \mathrm{~nm} / 458 \mathrm{~nm})$ were obtained by exciting $405 \mathrm{~nm}$ following stimulation with $10 \mu \mathrm{M}$ histamine. c, d Reduced $\mathrm{Ca}^{2+}$ load in both axonal and dendritic mitochondria in Mul1deficient neurons. The pseudo colors cyan $(458 \mathrm{~nm})$ and green $(525 \mathrm{~nm})$ represent the emission spectra of the ratiometric probe when excited with 405 $\mathrm{nm}$. The images were captured from dendritic and axonal compartments as indicated. The mitochondrial $\mathrm{Ca}^{2+}$ load was expressed as a ratio $(525 \mathrm{~nm} / 458$ $\mathrm{nm}$ ). Note that expressing Mul1 $\Delta$ Ring significantly reduces the ratio while expressing Mul1 enhances the ratio. Data were analyzed from 2 to 3 live neurons per field with 4 fields per experiment (b), or total number of fixed neurons indicated in the bars (d) in three experiments and expressed as mean \pm s.e.m. Unpaired Student's t-test was used for comparing two groups and Ordinary one-way ANOVA with Dunnett multiple comparison test for multiple comparisons. Scale Bars: $10 \mu \mathrm{m}$

stress conditions ${ }^{54,55}$, and was also observed in cells exposed to starvation $^{56}$, hypoxia ${ }^{57}$, cold stress ${ }^{58}$, and toxic insults ${ }^{59}$. It will be interesting to investigate into the role of Mul1-Mfn2 pathway in the maintenance and recovering of neuronal mitochondrial integrity in response to these conditions. Post-translational modifications, including phosphorylation and SUMOylation, regulate Drp1 activity under various stress conditions. In non-neuronal cells, Mul1 SUMOylates Drp1 in response to 
a

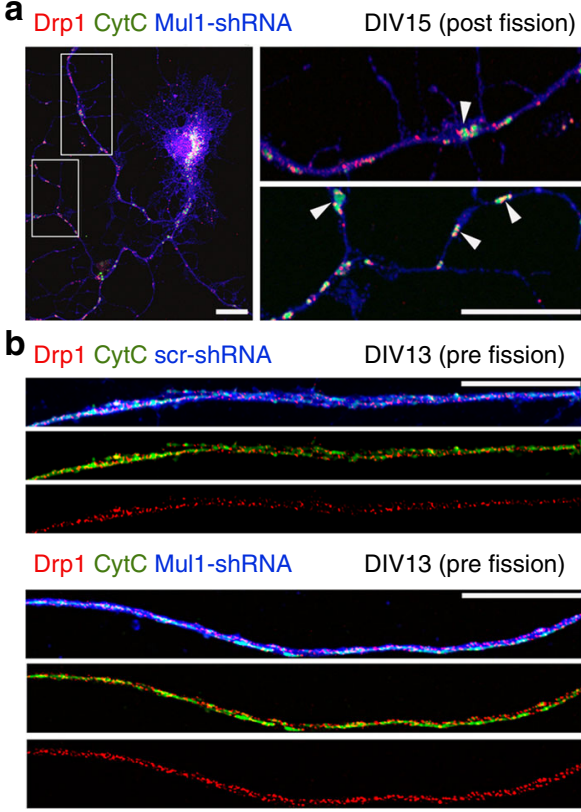

C
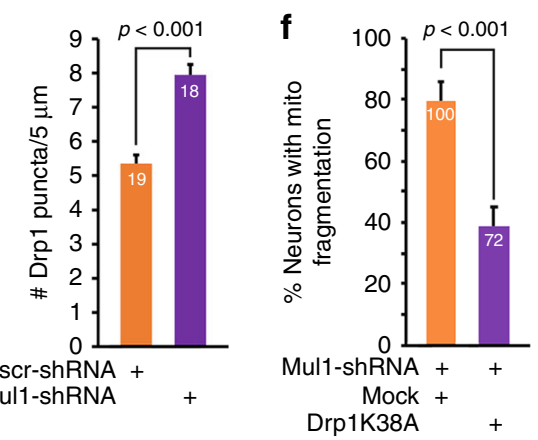

d
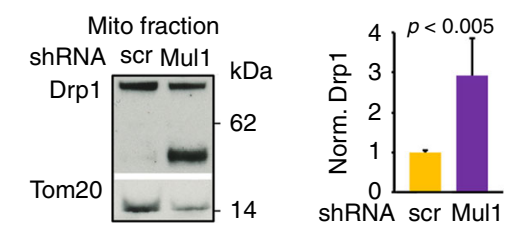

e
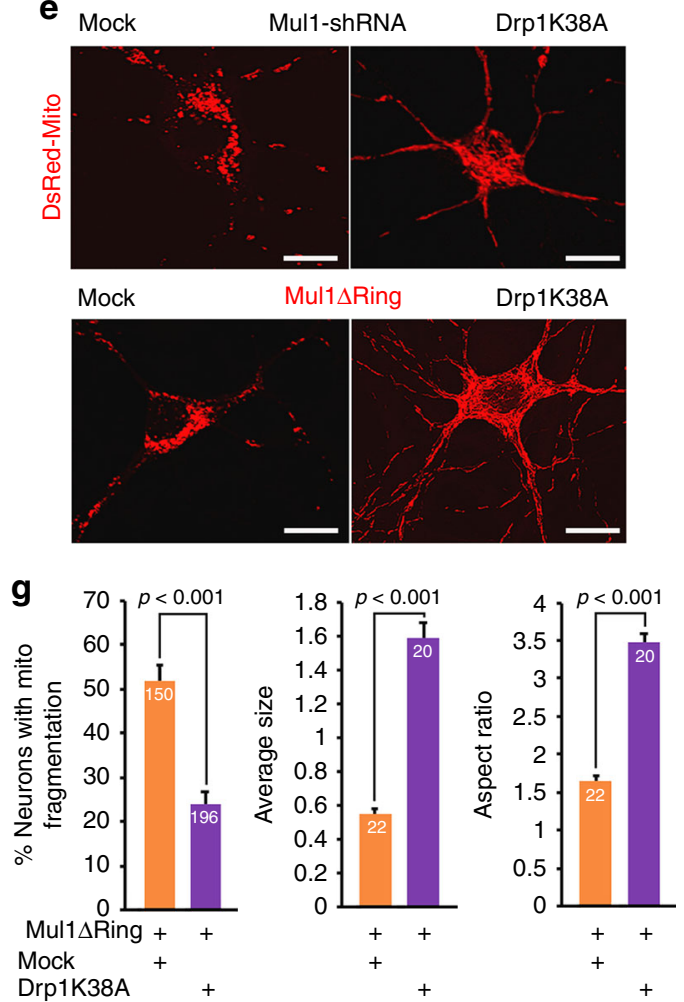

Fig. 7 Drp1 mediates second phasic mitochondrial fragmentation. a-c Representative images showing the targeting of Drp1 to fragmented mitochondria in Mul1-deficient neurons. Cortical neurons at DIV7-8 were co-transfected with GFP and Mul1-shRNA or scr-shRNA, followed by co-immunostaining of Drp1 (red) and cyto c (green) at DIV14-15 (a) or DIV13 (b) (pre-fission status). GFP (pseudo blue) was used to trace transfected neurons. Arrows point to Drp1 puncta on fragmented mitochondria (a). Average number of Drp1 puncta per $5 \mu \mathrm{m}$ mitochondrial length was measured from the total number of mitochondria (scr-shRNA, $n=518$; Mul1-shRNA, $n=423$ ) in the total number of neurons indicated in the bars. $\mathbf{d}$ A representative immunoblot showing an increased Drp1 translocation to mitochondria in Mul1 depleted neurons. Equal amounts ( $15 \mu \mathrm{g}$ ) of mitochondrial fractions were sequentially immunoblotted on the same membrane with each antibody after stripping. Note that a shorter Drp1 isoform ( $58 \mathrm{kDa})$ was recruited into mitochondrial fraction in Mul1deficient neurons. e-g Drp1-dependent mitochondrial fragmentation in Mul1-deficient neurons. Cortical neurons at DIV7-8 were co-transfected with Mul1shRNA, DsRed-Mito and Myc-tagged plasmid (Mock) or Drp1K38A, or co-transfected with Flag-tagged Mul1 Ring and Mock or Drp1K38A, followed by fixation and imaging at DIV14-15. Note that expressing Drp1K38A locks mitochondrial in hyperfusion status in the late stage of Mul1-deficient neurons. All data were analyzed from the total number of neurons indicated in the bars in three experiments and are expressed as mean \pm s.e.m. Unpaired Student's $t$-test. Scale Bars: $10 \mu \mathrm{m}$

severe apoptosis induction and thus stabilizes Drp1 on mitochondria ${ }^{17,18}$. However, in Mul1-deficient neurons without exogenous insult, we did not observe any significant change in mitochondrial SUMOylation levels.

Mfn2 is involved in multiple pathways in addition to mitochondrial fusion ${ }^{60}$. Overexpressing Mfn2 induces mitochondrial dysfunction and cell death ${ }^{61,62}$. Specifically, Mfn2 is involved in the regulation of ER-Mito contacts in MEFs and HeLa cells ${ }^{63}$. Mfn2 ablation in MEFs increases the distance between the ER and mitochondria and reduces mitochondrial $\mathrm{Ca}^{2+}$ uptake ${ }^{64}$. It was also reported that $\mathrm{Mfn} 2$ is required for mitochondrial $\mathrm{Ca}^{2+}$ uptake in skeletal muscle ${ }^{65}$ and cardiomyocytes ${ }^{66}$. This model was recently challenged by several studies. Using quantitative EM analysis, one study reported an increase, not a decrease, in the close contacts between ER and mitochondria in $M f n 2^{-/-}$cells ${ }^{19}$. In a second study, cells with deleted or reduced Mfn2 display increased ER-Mito tethering, enhanced $\mathrm{Ca}^{2+}$ transfer from ER to mitochondria, and increased sensitivity to apoptotic stimuli by mitochondrial $\mathrm{Ca}^{2+}$ overload $^{20}$. These ultrastructural and functional observations suggest an alternative model in which Mfn2 negatively modulates ER-Mito contacts, probably through its role as a tethering antagonist ${ }^{20,67,68}$. Such a role is likely mediated by lateral interference of increased Mfn2 with other linker components in the same membrane $\mathrm{e}^{22}$; this tethering antagonist may be more robust in neuronal ER-Mito contacts. Our current study in mature cortical neurons shows a negative role of $\mathrm{Mfn} 2$ in maintaining ER-Mito contacts. Using STED nanoscopy and iTEM ultrastructural analysis combined with mitochondrial $\mathrm{Ca}^{2+}$ uptake assay, our study consistently supports a role of Mull in sustaining ER-Mito contacts by reducing Mfn2 levels. Enhancing ER-Mito contacts by expressing an ER-Mito anchoring protein in Mul1-deficient neurons rescues mitochondrial fragmentation and suppresses Parkin-mediated mitophagy. Thus, maintaining ERMito contacts in mature neurons is critical to suppressing 
a

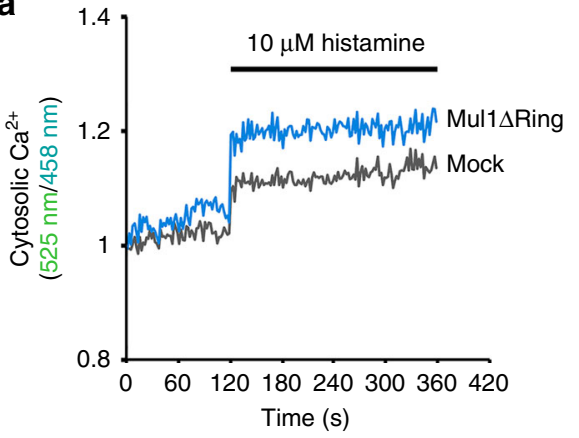

b

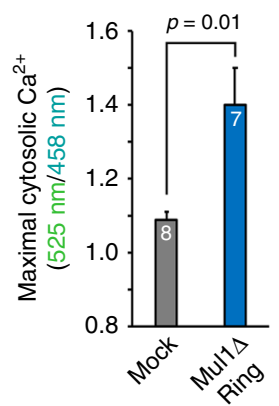

C

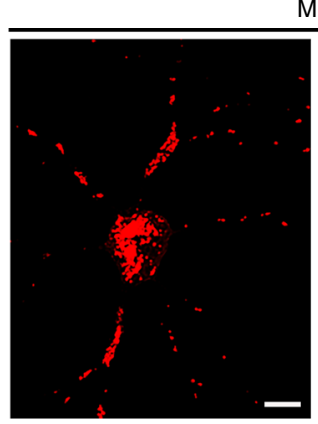

DMSO
Mul1-shRNA DsRed-Mito DIV14

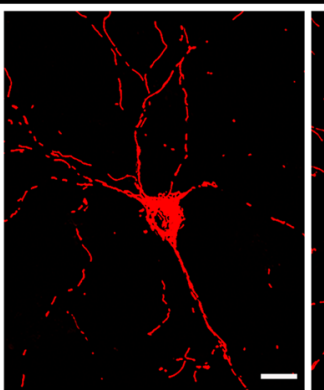

CsA

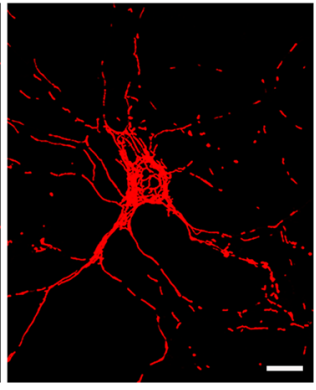

FK506 d

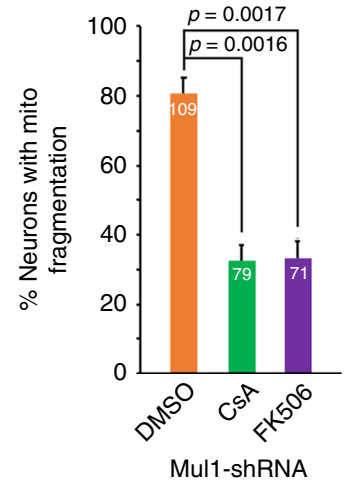

Fig. 8 Increased cytoplasmic $\mathrm{Ca}^{2+}$ load induces mitochondrial fragmentation. $\mathbf{a}$, $\mathbf{b}$ Representative traces and quantitative analysis of the average

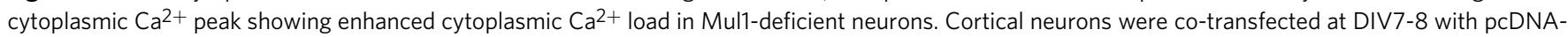
D3cpv and Flag or Flag-MUL1 $\Delta$ Ring, followed by live imaging at DIV12-13 just before mitochondrial fragmentation. The emission spectra of the ratiometric probe $(525 \mathrm{~nm} / 458 \mathrm{~nm})$ were obtained by exciting $405 \mathrm{~nm}$ following stimulation with $10 \mu \mathrm{M}$ histamine. Note that Mul1-deficient neurons display an increased cytoplasmic $\mathrm{Ca}^{2+}$ peak $\left(p=0.01\right.$ ) following histamine-induced $\mathrm{Ca}^{2+}$ release from the ER. Data were analyzed from 2 to 3 live neurons per field with 3 to 4 fields per experiment and the number of trials is indicated in the bars. c, $\mathbf{d}$ The role of calcineurin activity in mitochondrial fragmentation in Mul1-depleted neurons. Cortical neurons, co-transfected with DsRed-Mito and Mul1shRNA at DIV7-8, were treated with CsA (0.5 $\mu$ M) or FK506 (0.6 $\mu$ M) for $48 \mathrm{~h}$ starting at DIV12. Note that blocking calcineurin activity by CsA or FK506 abolishes mitochondrial fragmentation in Mul1-depleted neurons at DIV14. All data were analyzed from the total number of neurons indicated in the bars in three experiments and are expressed as mean \pm s.e.m. Unpaired Student's t-test (b) or Ordinary one-way ANOVA with Dunnett's post hoc test (d). Scale Bars: $10 \mu \mathrm{m}$

mitochondrial fragmentation and mitophagy. This notion is consistent with a recent report showing that impairing ERMito contacts increases Parkin-mediated mitophagy upon acute mitochondrial depolarization ${ }^{30}$. Parkin-mediated mitophagy is significantly delayed in mature primary neurons in response to mild mitochondrial stress ${ }^{6}$, a condition more relevant to pathological stress in aging-associated neurodegenerative diseases. Therefore, mitochondria-targeted Mull ideally acts as an early checkpoint via restraining Mfn2 levels and thus sustaining ERMito contacts. After this Mul1-Mfn2 pathway has failed, Parkin is recruited to mitochondria as the second resort for mitochondrial quality control. We further demonstrate that elevated Mfn2 activity impairs this protection mechanism, thus leading to rapid mitochondrial dysfunction and fragmentation. Our findings are supported by a recent study showing that altered Mfn2 activity has deleterious effects on neuronal mitochondrial function and is associated with neuropathy in Charcot-Marie-Tooth disease type 2A (CMT2A) ${ }^{69}$.

Several critical roles of mitochondria, including lipid and energy metabolism and $\mathrm{Ca}^{2+}$ homeostasis, rely on their interplay with the ER. Altered ER-Mito coupling has been reported in the pathogenesis of several major neurodegenerative diseases ${ }^{23,70}$. Although affected neurons in AD, PD, ALS/FTD, and HSP display various pathological changes, they all share common features: chronic mitochondrial dysfunction, altered ER-Mito interactions with impaired $\mathrm{Ca}^{2+}$ homeostasis and energy metabolism, and mitochondrial fragmentation ${ }^{23}$. Our study in mature cortical neurons reveals that sustaining ER-Mito contacts is critical to maintaining mitochondrial integrity, suppressing mitochondrial fragmentation and mitophagy, thus providing a mechanistic explanation for the seemingly disparate features of these neurodegenerative diseases.

\section{Methods}

Antibodies and DNA constructs. Sources of antibodies or reagents are as follows: antibodies against MAP2 (rabbit; AB5622; Millipore), p62/SQSTM1 (rabbit; PM045; MBL), Mul1 (rabbit; HPA017681; Sigma-Aldrich), Hsp60 (rabbit; 4870S; Cell Signaling), Lamp1 (rat; 1D4B; DSHB), HA (mouse; mms-101p; Covance), Mfn2 (rabbit; M6319; Sigma-Aldrich), Drp1 (rabbit; 8570; D6C7; Cell Signaling), Ser637-Drp1 (rabbit; 4867; Cell Signaling), mCherry (mouse; ab125096; abcam), Flag (mouse; F1804; Sigma-Aldrich, and rabbit; F7425; Sigma-Aldrich), GFP (rabbit; ab6556; abcam), TOM20 (rabbit; 11415; Santa Cruz), Ubiquitin (mouse, sc 8017; Santa Cruz), Myc (chicken; ab172; abcam), cytochrome c (mouse; 556432; BD Biosciences), Alexa Fluor 488- or 546- or 594- or 633-conjugated secondary antibodies (Invitrogen), Mouse IgG- or Rabbit IgG-HRP (GE HealthCare), and Nanogold (2004; Nanoprobe). The constructs GFP-Mul1, Flag-Mul1, GFP-Mul1H319A, and Mul1 shRNA were previously described ${ }^{16}$. Flag-tagged [Mul1 $\Delta$ Ring, Mul1 $\Delta(\mathrm{TM} 1 / \mathrm{TM} 2)]$ constructs were provided by C.A. Joazeiro (Scripps Inst., USA). pAc-GFPC1-Sec61beta, pCDNA3.1-Drp1(K38A), pcDNA-4mtD3cpv, pCDA-D3cpv, and Myc-tagged Mfn2(K109A) were purchased from Addgene Inc (Cambridge, MA). Construct for the ratiometric ATP sensor GO-ATeam2 was kindly provided by Hiromi Imamura (Kyoto University, Japan); Myc-tagged (Mfn1, Mfn2) constructs were provided by D. Chan (Caltech Inst., USA); MitoDsRed and m-Cherry Parkin constructs were provided by R. Youle (NINDS, NIH, USA). Mito-CFP was provided by D. C. Rubinstein (Cambridge University, UK). HA-PTPIP51 was provided by Christopher C.J. Miller (King's College, UK). 
a
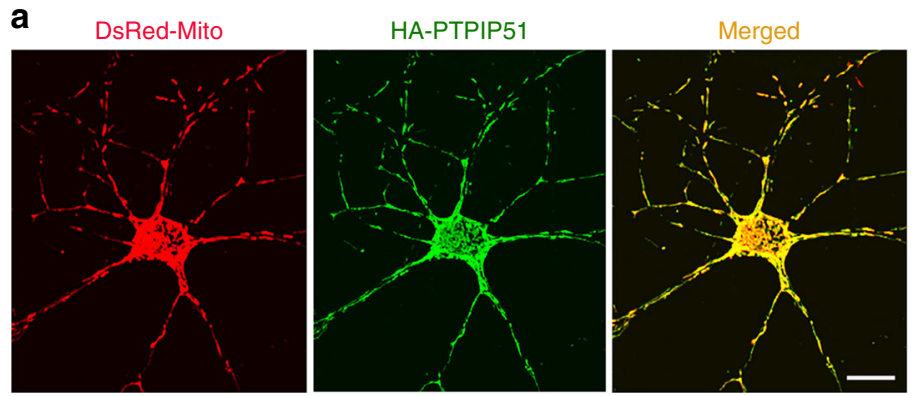

b

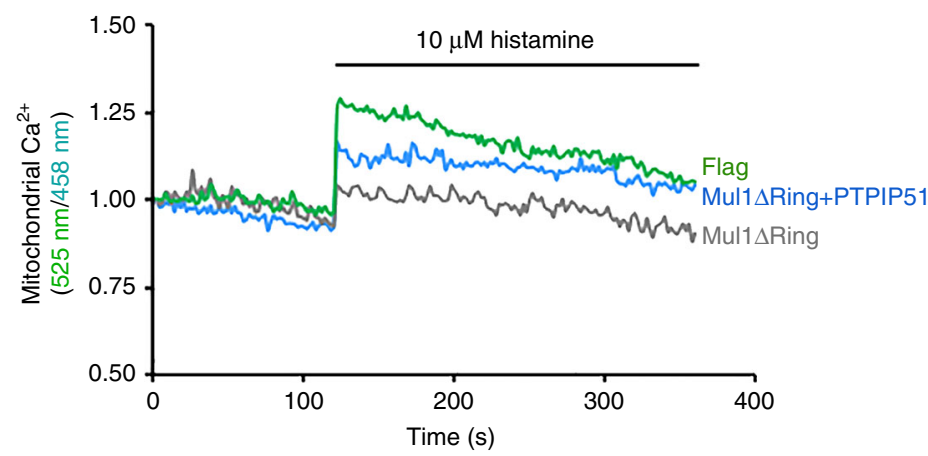

d

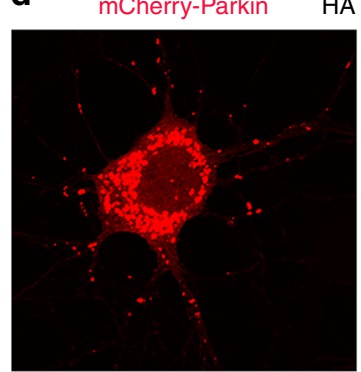

HA

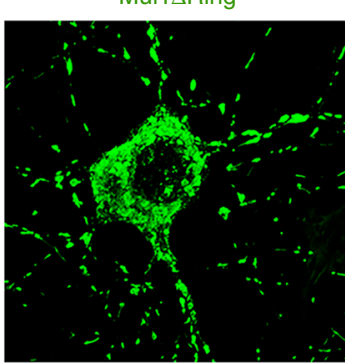

e

mCherry-Parkin HA-PTPIP51

Mul1 $\Delta$ Ring
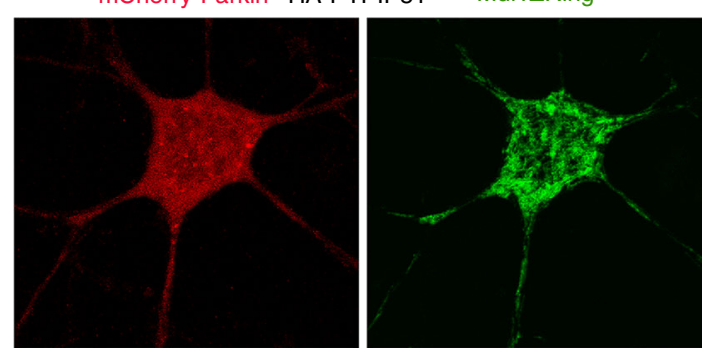

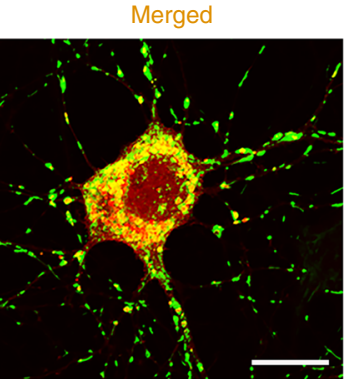

Merged

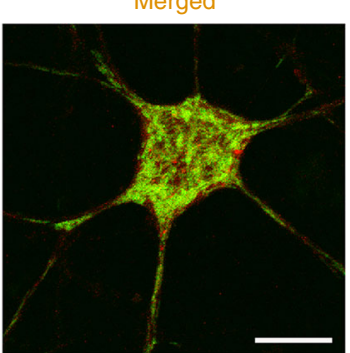

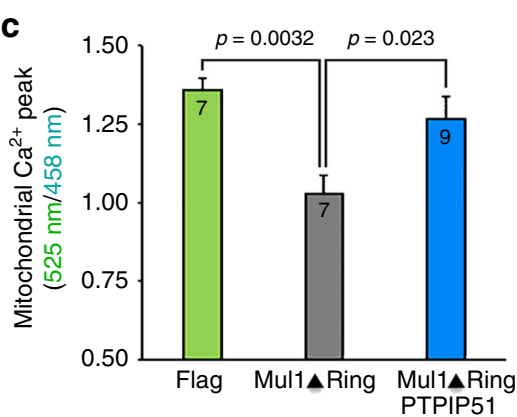
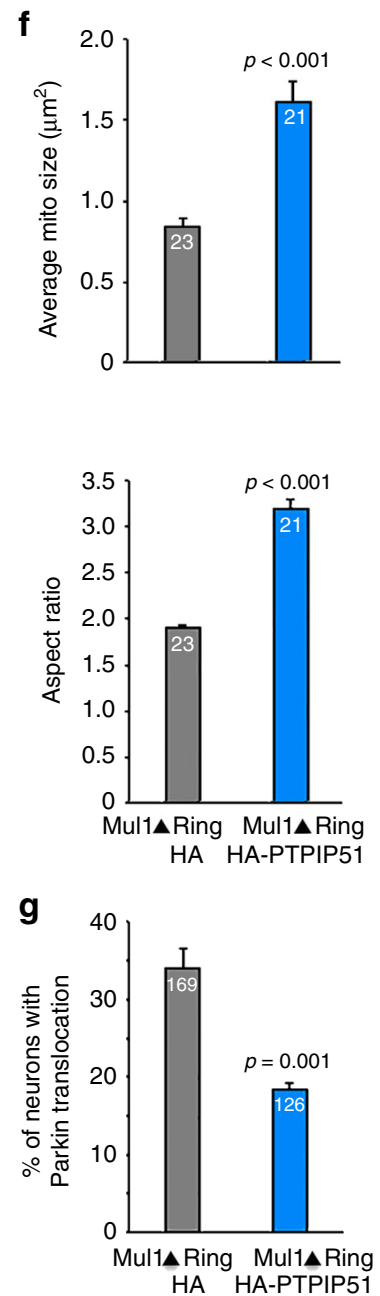

Fig. 9 Expressing PTPIP51 suppresses mitochondrial fragmentation and Parkin translocation in Mul1-deficient neurons. a Representative images showing the mitochondria-targeting of PTPIP51 in cortical neurons. Neurons were co-transfected with HA-tagged PTPIP51 and DsRed-Mito at DIV7-8 and imaged at DIV10-11 by immunostaining HA-tag. b, c Representative traces and quantitative analysis showing a partial rescue of IP3-induced ER-Mito Ca2+ exchange by expressing PTPIP51 in Mul1-deficient neurons. Cortical neurons at DIV7-8 were co-transfected with pcDNA-4mtD3cpv and Flag, or Flag-Mul1 4 Ring, or Flag-Mul1 $\Delta$ Ring + HA-PTPIP51. pcDNA-4mtD3cpv is a FRET-based ratiometric calcium probe that targets the mitochondrial matrix. Live neurons were imaged at DIV10-11. The traces represent the average fluorescence ratio signal ( $525 \mathrm{~nm} / 458 \mathrm{~nm}$ ) when excited with a $405-\mathrm{nm}$ laser following stimulation with $10 \mu \mathrm{M}$ histamine. Mitochondrial $\mathrm{Ca}^{2+}$ levels were measured from the total number of trials indicated in the bars. Each trial denotes 2-3 neurons per field. $\mathbf{d}-\mathbf{g}$ PTPIP51 expression suppresses mitochondrial fragmentation and reduces Parkin translocation in Mul1-deficient neurons. Cortical neurons at DIV7-8 were co-transfected with mCherry-Parkin, Flag-Mul14Ring, and HA-Vector (d) or HA-PTPIP51 (e), followed by immunostaining with Flag-tag (green) at DIV14-15. The mitochondrial morphology was measured as average mitochondria size and aspect ratio from the total number of neurons indicated in the bars (f); the percentage of neurons with Parkin recruitment to fragmented mitochondria was measured from the total number of neurons indicated in the bars $(\mathbf{g})$. All data were from three experiments using the unpaired Student's $t$-test $(\mathbf{f}, \mathbf{g})$ and Ordinary one-way ANOVA with Dunnett's post hoc test (c) and are expressed as mean \pm s.e.m. Scale Bars: $10 \mu \mathrm{m}$ 


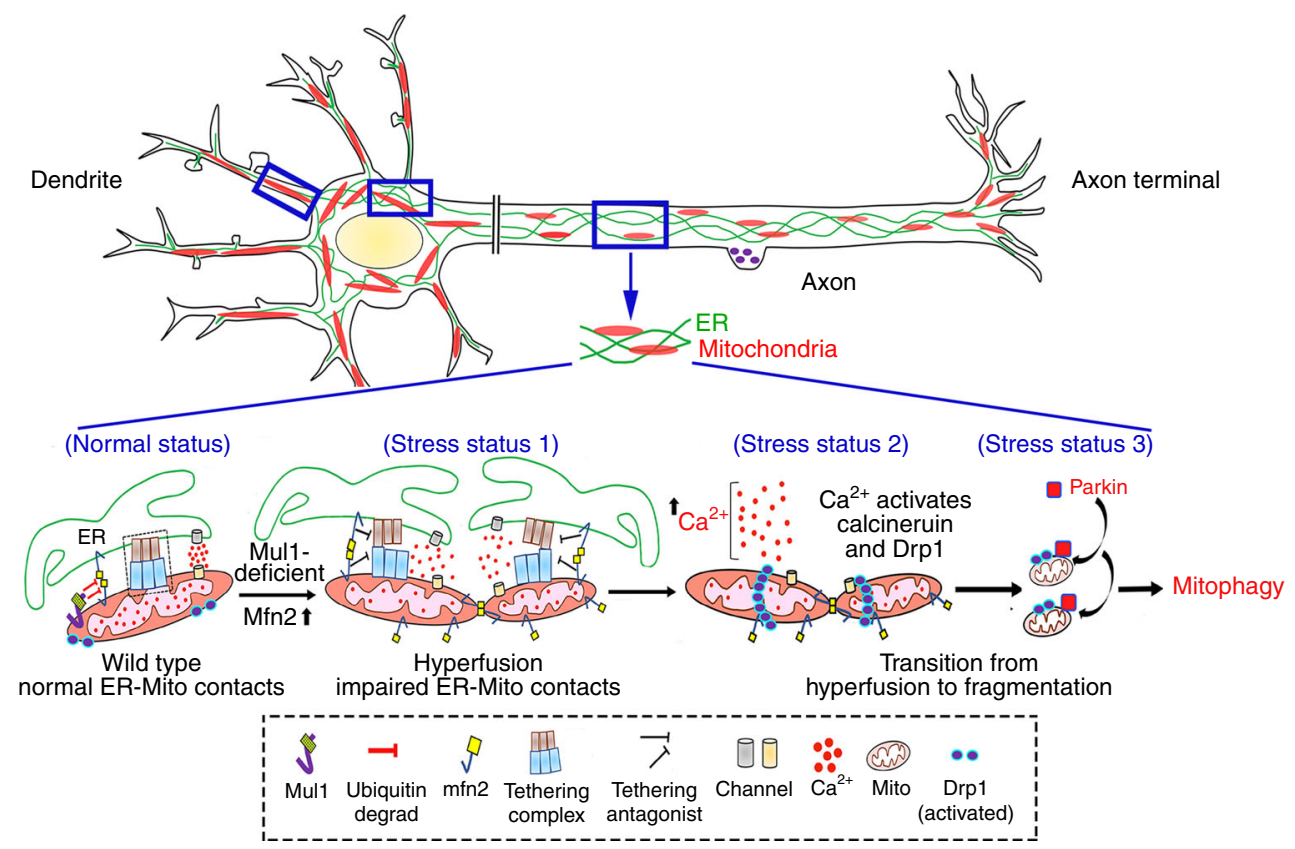

Fig. 10 Mul1-Mfn2 pathway in the maintenance of mitochondrial morphology and ER-Mito contacts. Mul1 acts as a mitochondrial E3 ubiquitin ligase that binds and ubiquitinates Mfn2, leading to Mfn2 degradation, thus maintaining mitochondrial morphology (normal status). Mul deficiency results in the first phasic mitochondrial hyperfusion by increasing Mfn2 activity (stress status 1). Increased Mfn2 activity parallelly acts as an ER-Mito tethering antagonist, thus impairing ER-Mito coupling, disturbing mitochondrial bioenergetics and $\mathrm{Ca}^{2+}$ homeostasis, and reducing mitochondrial $\mathrm{Ca}^{2+}$ uptake from the ER. An increased cytoplasmic $\mathrm{Ca}^{2+}$ load activates calcineurin phosphatase, which activates and recruits Drp1 onto stressed mitochondria (stress status 2), thus triggering second phasic mitochondrial fragmentation. Those fragmented mitochondria loss their membrane potential and ATP production capacity, thus recruiting Parkin for mitophagy (stress status 3). By parallel maintenance of mitochondrial morphology and ER-Mito contacts, Mul1-Mfn2 pathway plays a unique role in maintaining and/or recovering stressed mitochondria in neurons. If this protection pathway fails, mitophagy is activated to eliminate damaged mitochondria

Mt-Keima was purchased from MBL (\#AM-V0251HM) and verified by DNA sequencing. Mouse Mfn2 shRNA constructs were purchased from OriGene. The Mfn2 shRNA sequences are 5'-TCACCGTCAAGAAGGATAAGCGACACATG-3' and 5'-AGCAGAGCCAAACTGCTCAGGAATAAAGC-3'.

Cortical neuron culture and transfection. Cortices were dissected from E18-19 mouse embryos using the papain method. Cortical neurons were plated at a density of 0.5 million per $10 \mathrm{~cm}^{2}$ onto a 5-day-old glial feeder layer sitting on polyornithine (P4957; Sigma Aldrich) and matrigel (356234; BD Biosciences) coated coverslips. Neurons were grown overnight in plating medium with $5 \% \mathrm{FBS}$, insulin $(30 \mu \mathrm{g} / \mathrm{ml})$, GlutaMAX (35050-61, Thermo Fisher), and B27 (17504-044; Thermo Fisher) in Neurobasal medium (21103-049; Thermo Fisher). Starting at DIV2, cultures were maintained in conditioned medium with half-feed changes of neuronal feeding media every 3 days. Unless otherwise noted, neurons were transfected at DIV7-8 using the modified $\mathrm{Ca}^{2+}$ phosphate method and imaged at DIV10-11 or DIV14-15. Animal care and use were carried out in accordance with all relevant ethical regulations and NIH guidelines for animal testing and research. The study received ethical approval by the NIH, NINDS/NIDCD Animal Care and Use Committee.

Lentivirus production and infection. HEK293T cells were transfected with the vector, psPAX2, and pMD2G at a 4:3:1 ratio to produce the lentivirus expressing scramble or Mull shRNA. After a 24-hr transfection, the medium was replaced with $7 \mathrm{ml}$ of DMEM $+10 \%$ FBS. Virus-containing media were collected at 48 and $72 \mathrm{~h}$ post transfection and then centrifuged at $1600 \mathrm{rpm}$ for $5 \mathrm{~min}$ to remove cell debris. The pre-cleaned supernatant was collected and ultra-centrifuged at $90,000 \times g$ for $1.5 \mathrm{~h}$. The supernatant was carefully removed, and PBS was added to resuspend the viral pellets. The concentrated virus was aliquoted and stored at $-80^{\circ} \mathrm{C}$ until use. $1 \times 10^{7}$ of freshly dissociated cortical neurons were infected with a concentrated lentivirus.

Immunocytochemistry. Neurons were fixed at DIV10-11 or DIV14-15 with $4 \%$ paraformaldehyde (15710, Electron Microscopy System) and $4 \%$ sucrose in $1 \times$ phosphate-buffered saline (PBS) at $4{ }^{\circ} \mathrm{C}$ for 30 min or with $100 \%$ ice-cold methanol at $-20^{\circ} \mathrm{C}$ for $10 \mathrm{~min}$, followed by a 3-time wash with PBS for $5 \mathrm{~min}$ each, and incubated in PBS-based buffer containing 0.2\% saponin (S4521; Sigma-Aldrich), $5 \%$ goat serum, and $2 \%$ BSA for $1 \mathrm{~h}$. Fixed cultures were incubated with primary antibodies in PBS-based buffer containing $1 \%$ BSA and $0.05 \%$ saponin at $4{ }^{\circ} \mathrm{C}$ overnight. Samples were washed four times with PBS at room temperature (RT) for
5 min each, incubated with secondary fluorescent antibodies for $1 \mathrm{~h}$ at RT, rewashed with PBS, and then mounted with Fluoro-Gel anti-fade mounting medium (17985-10; Electron Microscopy System) for imaging.

Measurement of mitochondria morphology. Mitochondria were imaged after coexpressing Mito-DsRed with Mull or Mull mutants using an Olympus confocal microscope with an oil-immersion $\times 63$ objective/(NA-1.45). Unless otherwise noted, images were sequentially acquired below saturation from top to bottom with Z-step $(0.37 \mu \mathrm{m})$ at a resolution of $1024 \times 1024$ pixels, 12 bit, and $\times 2$ optical zoom. For mitochondrial size quantification, images were imported into ImageJ (NIH), converted to 8 bit, stacked using Z-projection (maximum intensity), and the background was removed using the ImageJ built-in plug-in "remove background". The images were thresholded, despeckled, and analyzed using the ImageJ (NIH) built-in plug-in "shape descriptor". The data were collected and transferred to Excel for analysis.

Mitochondria depolarization assay. Cortical neurons were treated for $3 \mathrm{~h}$ with DMSO or $100 \mathrm{nM}$ antimycin-A (AA) (A8674; Sigma-Aldrich) in combination with lysosomal inhibitors [LI; $10 \mu \mathrm{M}$ E64D (330005; EMD Millipore) and $10 \mu \mathrm{M}$ pepstatin A (El10, EMD Millipore)].

Inhibition of calcineurin activity. Cortical neurons were treated for $48 \mathrm{~h}$ with DMSO or $0.5 \mu \mathrm{M}$ cyclosporine A (CsA) (1101; Tocris Bioscience) or $0.6 \mu \mathrm{M}$ FK506 (F4679; Sigma-Aldrich).

Seahorse analysis. Oxygen consumption rate (OCR) was measured using a Seahorse XFe96 Flux Analyzer (Agilent Technologies). Briefly, cortical neurons were seeded in pentaplicate at $8 \times 10^{6}$ cells/well in an XF96 culture microplate and infected with scr-shRNA or Mul1-shRNA. Assays were initiated by removing the growth medium form each well and replacing it with $180 \mu \mathrm{l}$ of assay medium $(5 \mathrm{M}$ $\mathrm{NaCl}, 2 \mathrm{M} \mathrm{KCl}, 2 \mathrm{M} \mathrm{CaCl}_{2}, 1 \mathrm{M} \mathrm{KH}_{2} \mathrm{PO}_{4}, 2 \mathrm{M} \mathrm{MgCl}_{2}, 1 \mathrm{M}$ HEPES, $2.5 \mathrm{~m}$ glucose, $\mathrm{pH}$ 7.4) prewarmed to $37^{\circ} \mathrm{C}$. The cells were incubated at $37^{\circ} \mathrm{C}$ for $45 \mathrm{~min}$ to allow for media temperature and $\mathrm{pH}$ to reach equilibrium before the first-rate measurement. OCR measurements of mitochondrial function was obtained by sequential injections of $1 \mu \mathrm{M}$ oligomycin, $1 \mu \mathrm{M}$ FCCP, and $0.5 \mu \mathrm{M}$ rotenone/AA. All OCR measurements were normalized to non-mitochondrial respiration and the final values normalized to total cell number plated in each well as live cell numbers were not different between each group. 
Measurement of mitochondria $\Delta \boldsymbol{\Psi}_{\mathbf{m}}$. Neurons in conditioned growth media were incubated at $37^{\circ} \mathrm{C}$ with TMRE $(50 \mathrm{nM})\left(\mathrm{T} 669\right.$; Thermo Fisher) for $20 \mathrm{~min}$ in a $\mathrm{CO}_{2}$ incubator (Thermo Scientific). Post-treatment, neurons were transferred to imaging media containing (in mM) $20 \mathrm{HEPES}, 2 \mathrm{C}_{3} \mathrm{H}_{3} \mathrm{NaO}_{3}, 2$ GlutaMAX, with $2 \%$ B27 and $0.6 \%$ glucose in HBSS with $\mathrm{Ca}^{2+}$ and $\mathrm{Mg}^{2+}$ (14025-092; Thermo Fisher), washed 3 times with imaging media, and mounted for live-cell imaging. Confocal images were acquired within $30 \mathrm{~min}$ using an Olympus confocal oil-immersion $\times 63$ (1.45 NA) objective with image acquisition settings set below saturation. Five to six optical sections for each image were taken from top-to-bottom of the specimen. Measurement of TMRE integrated intensity was done using Image J and expressed as corrected total cell fluorescence (CTCF) using the formula (CTCF $=$ Integrated mean intensity - area mean background values). The mean fluorescent intensity of TMRE in the soma for each transfected group was normalized as a percentile ratio relative to that in neurons expressing the GFP construct.

ATP measurement. A FRET-based fluorescent ATP probe (GO-ATeam2) was used to measure intracellular cytosolic ATP levels ${ }^{38}$. Briefly, the probe encodes FRET pair fluorescent proteins, green fluorescent protein (GFP), and orange fluorescent protein (OFP). Transfected neurons were visualized with an Olympus Fluoview (F1000) inverted microscope (Olympus Corp., USA) using the Plan Fluor $\times 63$ (1.45 NA) oil-immersion objective lens (Olympus). The filters used for dualemission ratio imaging of GO-ATeam were purchased from Semrock (Rochester, NY) and included an FF01-482/18 excitation filter, an FF495-DiO2 dichroic mirror, and two emission filters (FF01-520/35 for GFP and FF01-562/40 for OFP). The images were imported to ImageJ (NIH); CTCF values for each channel (GFP and OFP) were quantified and the ratio (OFP/GFP) was calculated using Microsoft Excel software.

Immuno-electron microscopy. Wild-type or Mul1-deficient neurons expressing a GFP-tagged ER marker (Sec61ß) were fixed at DIV10-11 in a PBS-based mix of $4 \%$ paraformaldehyde and $0.1 \%$ glutaraldehyde (16020; Electron Microscopy System) for $30 \mathrm{~min}$ at RT. The samples were washed with PBS four times, blocked and permeabilized with a PBS-based mix of 5\% normal goat serum and $0.1 \%$ saponin for $1 \mathrm{~h}$ at RT. Samples were then incubated with a primary antibody [anti-GFP $(1: 500)]$ in a PBS-based buffer containing 5\% normal goat serum and $0.05 \%$ saponin for $1 \mathrm{hr}$ at RT, washed with PBS four times, and incubated with nanogold Fab' conjugates (1:200 dilution) (Nanoprobe) made in PBS containing 5\% normal goat serum and $0.05 \%$ saponin for another hour at RT. Samples were then washed with PBS, fixed with $2 \%$ glutaraldehyde in PBS for 30 min, silver enhanced with $0.2 \% \mathrm{OsO}_{4}$ in $0.1 \mathrm{M}$ phosphate buffer for $30 \mathrm{~min}$, and en bloc mordanted with uranyl acetate. The samples were then dehydrated in a series of graded ethanol washes and embedded in epoxy resins. Thin sections were stained with uranyl acetate and lead citrate (EM Facility, National Institute of Neurological Disorders and Stroke, National Institutes of Health). Sections were visualized under a JEOL (Akshima, Japan) 1200 EX transmission electron microscope (TEM) and digital images were captured with a CCD camera system (XR-100; Advanced Microscopy Techniques, Danver, MA). The TEM images were analyzed using ImageJ.

Super-resolution 3D-STED imaging. Dual-color 3D-STED imaging was performed by using Leica TCS SP8 STED 3X, Germany. For fixed-cell imaging, the fluorophores were excited with a tunable white light laser $(70 \%$ of maximum power). The images were captured using two HyD detectors and an oil objective (HC PLAPO 100X/1.40 OIL). For imaging GFP, cells were excited at $488 \mathrm{~nm} \mathrm{(25 \% )}$ with the STED depletion laser at $592 \mathrm{~nm}(15 \%)$; its emission fluorescence at $495-584 \mathrm{~nm}$ was collected using time-gated HyD detectors (1.25-6.5 ns). Alexa 594 were excited at $590 \mathrm{~nm}(1.25 \%)$ with the STED depletion laser at $660 \mathrm{~nm}(10 \%)$; its emission fluorescence between 594-660 nm was collected using time-gated HyD detectors (1.25-6.5 ns). For dual-color imaging of Alexa Fluor 594 and GFP 488, Alexa Fluor 594 was first imaged to avoid bleaching by the $592 \mathrm{~nm}$ STED depletion laser in the frame-scanning mode. Image acquisition and the microscope platform were controlled by built-in Leica Application Suite (LASX) software. For image acquisition, settings were kept below saturation at $1028 \times 1028$ pixels, 8 bit, with a $(\times 5)$ optical zoom for capturing the soma-dendritic region and a $(\times 6)$ optical zoom for capturing dendrites. Images were acquired sequentially from top to bottom using system-optimized Z-step setting and then were deconvolved using a Leica built-in Huygens STED deconvolution software.

Preparation of samples for STED. A modified fixation protocol was used to improve an ER fixation procedure for STED. Mouse cortical neurons were fixed post-transfection at DIV10-11 by briefly rinsing in a $2 \%$ sucrose solution in PBS, followed by dipping in tetrahydrofuran and being kept on dry ice overnight. The next day, the coverslips were placed for fixation in a solution of $10 \mathrm{ml}$ methanol and $1 \mathrm{ml} 16 \%$ paraformaldehyde and kept at $-20^{\circ} \mathrm{C}$ for $3 \mathrm{~h}$. Coverslips were transferred post-fixation to RT for $1 \mathrm{~h}$. Samples were rehydrated in graded concentrations of ethanol in PBS, starting with $100 \%-95 \%-70 \%-50 \%$, for 10 min each. Post-fixation, cells were immunostained for mitochondria using an antibody against Tom $20(1: 100)$ overnight at $4{ }^{\circ} \mathrm{C}$, followed by treatment with a 594conjugated secondary antibody (1:50) for $1 \mathrm{~h}$ at RT. High-resolution images for each transfected group were captured using Leica TCS SP8 STED 3X. Meander's colocalization analysis between mitochondria and ER was done using a built-in colocalization plug-in in Velocity Software. The data were transferred to Excel for analysis.

Ubiquitination assay. Cortical neurons infected with scramble or Mul shRNA were treated with $10 \mathrm{uM}$ MG132 (474790; Calbiochem) for $4 \mathrm{~h}$. Cells were then lysed in RIPA lysis buffer (50 mM Tris, pH 7.5, $150 \mathrm{mM} \mathrm{NaCl}, 1 \%$ Triton X-100, $0.1 \%$ SDS, and $1 \%$ sodium deoxycholate) supplemented with protease inhibitor. The lysate was then incubated with control rabbit IgG (2729S; Cell Signaling) or anti-Mfn2 antibody (9842; Cell Signaling) at $4^{\circ} \mathrm{C}$ overnight, and then incubated with protein A-Sepharose (17-0780-01; GE-Healthcare). The recovered immunocomplex was extensively washed with lysis buffer four times and eluted with reducing LDS sample buffer. Samples were resolved with 4-12\% NuPAGE followed by immunoblotting with mouse anti-Ub (sc-8017; Santa Cruz), and rabbit antiMfn2 (11925; Cell Signaling).

Isolation of mitochondrial fraction. Cortical neurons infected with scramble or Mull shRNA were washed 2 times with PBS and scrapped over ice with mitochondrial isolation buffer $(220 \mathrm{mM}$ mannitol, $80 \mathrm{mM} \mathrm{KCl}, 0.5 \mathrm{mM}$ EGTA, $20 \mathrm{mM}$ HEPES, $68 \mathrm{mM}$ sucrose, $2 \mathrm{mM} \mathrm{MgCl}, 20 \mathrm{mM} \mathrm{N}$-ethyl maleimide and protease inhibitors). Cell suspension were broken by passing through a 26-gauge needle. Samples were then centrifuged at $800 \times g$ at $4{ }^{\circ} \mathrm{C}$ for $10 \mathrm{~min}$ to pellet the nuclear fraction. The supernatants were collected and centrifuged at $800 \times g$ at $4{ }^{\circ} \mathrm{C}$ for $5 \mathrm{~min}$ to completely remove the nuclear fraction. Post-nuclear supernatants were then centrifuged at $9000 \times g$ at $4^{\circ} \mathrm{C}$ for $20 \mathrm{~min}$ to pellet the mitochondrial fraction The mitochondrial pellet was then dissolved in RIPA buffer. The protein content was normalized to the mitochondrial fraction and processed for PAGE and western blotting.

Immunoblotting. Human embryonic kidney cells (HEK293T) were cultured in 35$\mathrm{mm}$ plates at a density of $\sim 1 \times 10^{6}$ and constructs were transfected using Lipofectamine 2000 (11668019, Thermo Fisher). For immunoblotting, samples were harvested and suspended in ice-cold RIPA lysis Buffer [ $150 \mathrm{mM} \mathrm{NaCl}, 0.1 \%$ Triton $\mathrm{X}-100,0.5 \%$ sodium deoxycholate, $0.1 \%$ SDS, $50 \mathrm{mM}$ Tris- $\mathrm{HCl} \mathrm{pH} \mathrm{8.0,} \mathrm{protease}$ inhibitor (P8430; Sigma Aldrich) and phosphatase inhibitor cocktail (4906845001; Sigma Aldrich). The lysates were centrifuged at $13,000 \times g$ for $15 \mathrm{~min}$ at $4^{\circ} \mathrm{C}$; pellets were discarded; and the supernatant was assayed for protein concentration The protein $(5 \mu \mathrm{g})$ was resolved on $4-12 \%$ Bis-Tris gels (12313623; Invitrogen) followed by Western blots on the same membranes after stripping between each application of the antibody.

$\mathrm{Ca}^{2+}$ measurements. Two FRET-based ratiometric fluorescent $\mathrm{Ca}^{2+}$ probes were used to measure $\mathrm{Ca}^{2+}$ : pCDNA-D3cpv that targets the cytoplasm and pcDNA$4 \mathrm{mtD} 3 \mathrm{cpv}$ that targets the mitochondrial matrix ${ }^{44}$. Briefly, the probe encodes FRET pair fluorescent proteins, an enhanced cyan fluorescent protein (CFP), and circularly permuted Venus 173 . The probe was transfected in primary neuron cells by the $\mathrm{Ca}^{2+}$ phosphate method at DIV7-8 and imaged at DIV10-11. Samples were treated with $10 \mu \mathrm{M}$ Histamine (H7125; Sigma-Aldrich) for releasing $\mathrm{Ca}^{2+}$ from the ER. Samples were visualized with an Olympus Fluoview (F1000) inverted microscope (Olympus Corp., USA) using the Plan Fluor 63x/(1.45 NA), oil-immersion objective lens (Olympus). Filters used for dual-emission ratio imaging of pCDNA D3cpv and pcDNA-4mtD3cpv were purchased from Semrock (Rochester, NY). Images were imported to ImageJ.

Measurement of mitophagy with mt-Keima. For cortical neurons co-transfected with $\mathrm{mt}$-Keima and other different constructs, live-cell imaging was performed in Low Fluorescence Hibernate A media (BrainBits) with $0.5 \mathrm{mM}$ Glutamax and B27, and maintained in an environmental chamber where the temperature was kept at $37^{\circ} \mathrm{C}$. Neurons were visualized with a Zeiss LSM 880 oil immersion $63 \mathrm{x}$ objective with two sequential excitation lasers at $458 \mathrm{~nm}$ (green, healthy mitochondria at neutral $\mathrm{pH}$ ) and 561 (red, damaged mitochondria engulfed by lysosomes under acidic $\mathrm{pH}$ ) and an emission spectrum from $590-680 \mathrm{~nm}$. Images were sequentially acquired from top to bottom with Z-step $(1 \mu \mathrm{m})$ at a resolution of $1024 \times 1024$ pixels, 8 bit. Images were imported into Image (NIH), stacked using Z-projection (maximum intensity), and the background was removed using the built-in plugin "remove background". With post-processed images, mitophagy index was defined as the ratio of the area of lysosomal signal (red)/mitochondrial signal (green) in the cell body, and were normalized to control conditions.

Quantification and statistical analysis. All quantifications were performed unblinded. Statistical parameters including the definitions and exact value of $\mathrm{n}$ (e.g., total number of experiments, replications, axons, organelles, or neurons), deviations, $\mathrm{p}$ values, and the types of the statistical tests are reported in the figures and corresponding figure legends. Statistical analysis was carried out using Prism 7 (GraphPad Software). Statistical analysis was conducted on data from three or more biologically independent experimental replicates. Comparisons between groups were planned before statistical testing and target effect sizes were not predetermined. Error bars displayed on graphs represent the mean \pm SEM of at 
least three independent experiments. Statistical significance was analyzed using unpaired Student's $t$-test for two groups or Ordinary one-way ANOVA with Dunnett multiple comparison test for multiple groups. ${ }^{*} p<0.05$, ${ }^{* *} p<0.01$, and ${ }^{* * *} p<0.001$ were considered significant.

Reporting summary. Further information on research design is available in the Nature Research Reporting Summary linked to this article.

\section{Data availability}

The data sets generated and/or analyzed during the current study are available from the corresponding author on request.

Received: 8 November 2017 Accepted: 27 July 2019

Published online: 13 August 2019

\section{References}

1. Mishra, P. \& Chan, D. C. Metabolic regulation of mitochondrial dynamics. J. Cell Biol. 212, 379-387 (2016).

2. Nunnari, J. \& Suomalainen, A. Mitochondria: in sickness and in health. Cell 148, 1145-1159 (2012)

3. Sheng, Z.-H. \& Cai, Q. Mitochondrial transport in neurons: impact on synaptic homeostasis and neurodegeneration. Nat. Rev. Neurosci. 13, 77-93 (2012).

4. Pickrell, A. M. \& Youle, R. J. The roles of PINK1, parkin, and mitochondrial fidelity in Parkinson's disease. Neuron 85, 257-273 (2015).

5. Cai, Q., Zakaria, H. M., Simone, A. \& Sheng, Z.-H. Spatial parkin translocation and degradation of damaged mitochondria via mitophagy in live cortical neurons. Curr. Biol. 22, 545-552 (2012).

6. Lin, M.-Y. et al. Releasing syntaphilin removes stressed mitochondria from axons independent of mitophagy under pathophysiological conditions. Neuron 94, 595-610.e6 (2017).

7. Ashrafi, G., Schlehe, J. S., LaVoie, M. J. \& Schwarz, T. L. Mitophagy of damaged mitochondria occurs locally in distal neuronal axons and requires PINK1 and Parkin. J. Cell Biol. 206, 655-670 (2014).

8. Kitada, T., Tong, Y., Gautier, C. A. \& Shen, J. Absence of nigral degeneration in aged parkin/DJ-1/PINK1 triple knockout mice. J. Neurochem. 111, 696-702 (2009).

9. Perez, F. A. \& Palmiter, R. D. Parkin-deficient mice are not a robust model of parkinsonism. Proc. Natl Acad. Sci. USA 102, 2174-2179 (2005).

10. Whitworth, A. J. \& Pallanck, L. J. PINK1/Parkin mitophagy and neurodegeneration-what do we really know in vivo? Curr. Opin. Genet. Dev. 44, 47-53 (2017).

11. Devireddy, S., Liu, A., Lampe, T. \& Hollenbeck, P. J. The organization of mitochondrial quality control and life cycle in the nervous system in vivo in the absence of PINK1. J. Neurosci. 35, 9391-9401 (2015).

12. Sung, H., Tandarich, L. C., Nguyen, K. \& Hollenbeck, P. J. CompArtmentalized Regulation of Parkin-mediated mitochondrial quality control in the Drosophila nervous system in vivo. J. Neurosci. 36, 7375-7391 (2016).

13. Neuspiel, M. et al. Cargo-selected transport from the mitochondria to peroxisomes is mediated by vesicular carriers. Curr. Biol. 18, 102-108 (2008).

14. Li, W. et al. Genome-wide and functional annotation of human E3 ubiquitin ligases identifies MULAN, a mitochondrial E3 that regulates the organelle's dynamics and signaling. PLoS ONE 3, e1487 (2008).

15. Zhang, B. et al. GIDE is a mitochondrial E3 ubiquitin ligase that induces apoptosis and slows growth. Cell Res. 18, 900-910 (2008)

16. Yun, J. et al. MUL1 acts in parallel to the PINK1/parkin pathway in regulating mitofusin and compensates for loss of PINK1/parkin. Elife 3, e01958 (2014).

17. Braschi, E., Zunino, R. \& McBride, H. M. MAPL is a new mitochondrial SUMO E3 ligase that regulates mitochondrial fission. EMBO Rep. 10, 748-754 (2009).

18. Prudent, J. et al. MAPL sumoylation of drp1 stabilizes an er/mitochondrial platform required for cell death. Mol. Cell 59, 941-955 (2015).

19. Cosson, P., Marchetti, A., Ravazzola, M. \& Orci, L. Mitofusin-2 independent juxtaposition of endoplasmic reticulum and mitochondria: an ultrastructural study. PLoS ONE 7, e46293 (2012).

20. Filadi, R. et al. Mitofusin 2 ablation increases endoplasmic reticulummitochondria coupling. Proc. Natl Acad. Sci. USA 112, E2174-E2181 (2015).

21. Filadi, R. et al. Presenilin 2 modulates endoplasmic reticulum-mitochondria coupling by tuning the antagonistic effect of mitofusin 2. Cell Rep. 15, 2226-2238 (2016).

22. Csordás, G., Weaver, D. \& Hajnóczky, G. Endoplasmic reticulummitochondrial contactology: structure and signaling functions. Trends Cell Biol. 28, 523-540 (2018)
23. Paillusson, S. et al. There's something wrong with my MAM; the ERmitochondria axis and neurodegenerative diseases. Trends Neurosci. 39, 146-157 (2016)

24. Gomez-Suaga, P. et al. The ER-mitochondria tethering complex VAPBPTPIP51 regulates autophagy. Curr. Biol. 27, 371-385 (2017).

25. Bravo, R. et al. Increased ER-mitochondrial coupling promotes mitochondrial respiration and bioenergetics during early phases of ER stress. J. Cell Sci. 124, 2143-2152 (2011)

26. Danese, A. et al. Calcium regulates cell death in cancer: Roles of the mitochondria and mitochondria-associated membranes (MAMs). Biochim. Biophys. Acta Bioenerg. 1858, 615-627 (2017).

27. Ramírez, O. A. \& Couve, A. The endoplasmic reticulum and protein trafficking in dendrites and axons. Trends Cell Biol. 21, 219-227 (2011).

28. Mironov, S. L. \& Symonchuk, N. ER vesicles and mitochondria move and communicate at synapses. J. Cell Sci. 119, 4926-4934 (2006).

29. Krols, M., Bultynck, G. \& Janssens, S. ER-Mitochondria contact sites: a new regulator of cellular calcium flux comes into play. J. Cell Biol. 214, 367-370 (2016).

30. McLelland, G.-L. et al. Mfn2 ubiquitination by PINK1/parkin gates the p97dependent release of ER from mitochondria to drive mitophagy. Elife 7 , e32866 (2018)

31. Clark, I. E. et al. Drosophila pink1 is required for mitochondrial function and interacts genetically with parkin. Nature 441, 1162-1166 (2006).

32. Lim, Y., Cho, I.-T., Schoel, L. J., Cho, G. \& Golden, J. A. Hereditary spastic paraplegia-linked REEP1 modulates endoplasmic reticulum/mitochondria contacts. Ann. Neurol. 78, 679-696 (2015)

33. Krols, M. et al. Mitochondria-associated membranes as hubs for neurodegeneration. Acta Neuropathol. 131, 505-523 (2016).

34. Narendra, D., Tanaka, A., Suen, D.-F. \& Youle, R. J. Parkin is recruited selectively to impaired mitochondria and promotes their autophagy. J. Cell Biol. 183, 795-803 (2008).

35. Van Laar, V. S. et al. Bioenergetics of neurons inhibit the translocation response of Parkin following rapid mitochondrial depolarization. Hum. Mol. Genet. 20, 927-940 (2011).

36. Miller, K. E. \& Sheetz, M. P. Axonal mitochondrial transport and potential are correlated. J. Cell Sci. 117, 2791-2804 (2004).

37. Katayama, H., Kogure, T., Mizushima, N., Yoshimori, T. \& Miyawaki, A. A sensitive and quantitative technique for detecting autophagic events based on lysosomal delivery. Chem. Biol. 18, 1042-1052 (2011).

38. Nakano, M., Imamura, H., Nagai, T. \& Noji, H. $\mathrm{Ca}^{2+}$ regulation of mitochondrial ATP synthesis visualized at the single cell level. ACS Chem. Biol. 6, 709-715 (2011).

39. Zhou, B. et al. Facilitation of axon regeneration by enhancing mitochondrial transport and rescuing energy deficits. J. Cell Biol. 214, 103-119 (2016).

40. Chen, H. et al. Mitofusins Mfn1 and Mfn2 coordinately regulate mitochondrial fusion and are essential for embryonic development. J. Cell Biol. 160, 189-200 (2003).

41. Marchi, S., Patergnani, S. \& Pinton, P. The endoplasmic reticulummitochondria connection: one touch, multiple functions. Biochim. Biophys. Acta 1837, 461-469 (2014).

42. Csordás, G. et al. Imaging interorganelle contacts and local calcium dynamics at the ER-mitochondrial interface. Mol. Cell 39, 121-132 (2010).

43. De Vos, K. J. et al. VAPB interacts with the mitochondrial protein PTPIP51 to regulate calcium homeostasis. Hum. Mol. Genet. 21, 1299-1311 (2012).

44. Palmer, A. E. et al. $\mathrm{Ca}^{2+}$ indicators based on computationally redesigned calmodulin-peptide pairs. Chem. Biol. 13, 521-530 (2006).

45. Marcu, R., Wiczer, B. M., Neeley, C. K. \& Hawkins, B. J. Mitochondrial matrix $\mathrm{Ca}^{2+}$ accumulation regulates cytosolic $\mathrm{NAD}^{+} / \mathrm{NADH}$ metabolism, protein acetylation, and sirtuin expression. Mol. Cell. Biol. 34, 2890-2902 (2014).

46. Itoh, K. et al. A brain-enriched Drp1 isoform associates with lysosomes, late endosomes, and the plasma membrane. J. Biol. Chem. 293, 11809-11822 (2018).

47. Cereghetti, G. M. et al. Dephosphorylation by calcineurin regulates translocation of Drp1 to mitochondria. Proc. Natl. Acad. Sci. USA 105, 15803-15808 (2008).

48. Bernardi, P. Mitochondrial transport of cations: channels, exchangers, and permeability transition. Physiol. Rev. 79, 1127-1155 (1999).

49. Friberg, H., Ferrand-Drake, M., Bengtsson, F., Halestrap, A. P. \& Wieloch, T. Cyclosporin A, but not FK 506, protects mitochondria and neurons against hypoglycemic damage and implicates the mitochondrial permeability transition in cell death. J. Neurosci. 18, 5151-5159 (1998).

50. Palacino, J. J. et al. Mitochondrial dysfunction and oxidative damage in parkin-deficient mice. J. Biol. Chem. 279, 18614-18622 (2004).

51. Gispert, S. et al. Parkinson phenotype in aged PINK1-deficient mice is accompanied by progressive mitochondrial dysfunction in absence of neurodegeneration. PLoS ONE 4, e5777 (2009).

52. Schon, E. A. \& Przedborski, S. Mitochondria: the next (neurode)generation. Neuron 70, 1033-1053 (2011). 
53. Wideman, J. G. \& Moore, B. P. The evolutionary history of MAPL (mitochondria-associated protein ligase) and other eukaryotic BAM/GIDE domain proteins. PLoS ONE 10, e0128795 (2015).

54. Legros, F., Lombès, A., Frachon, P. \& Rojo, M. Mitochondrial fusion in human cells is efficient, requires the inner membrane potential, and is mediated by mitofusins. Mol. Biol. Cell 13, 4343-4354 (2002).

55. Chen, H., Chomyn, A. \& Chan, D. C. Disruption of fusion results in mitochondrial heterogeneity and dysfunction. J. Biol. Chem. 280, 26185-26192 (2005)

56. Rambold, A. S., Kostelecky, B., Elia, N. \& Lippincott-Schwartz, J. Tubular network formation protects mitochondria from autophagosomal degradation during nutrient starvation. Proc. Natl. Acad. Sci. USA 108, 10190-10195 (2011).

57. Chiche, J. et al. Hypoxic enlarged mitochondria protect cancer cells from apoptotic stimuli. J. Cell Physiol. 222, 648-657 (2010).

58. Zhang, W. et al. Mitofusin-2 protects against cold stress-induced cell injury in HEK293 cells. Biochem. Biophys. Res. Commun. 397, 270-276 (2010).

59. van der Bliek, A. M. Fussy mitochondria fuse in response to stress. EMBO J. 28, 1533-1534 (2009).

60. Schrepfer, E. \& Scorrano, L. Mitofusins, from mitochondria to metabolism. Mol. Cell 61, 683-694 (2016).

61. Huang, P., Yu, T. \& Yoon, Y. Mitochondrial clustering induced by overexpression of the mitochondrial fusion protein Mfn2 causes mitochondrial dysfunction and cell death. Eur. J. Cell Biol. 86, 289-302 (2007).

62. Shen, T. et al. Mitofusin-2 is a major determinant of oxidative stress-mediated heart muscle cell apoptosis. J. Biol. Chem. 282, 23354-23361 (2007).

63. de Brito, O. M. \& Scorrano, L. Mitofusin 2 tethers endoplasmic reticulum to mitochondria. Nature 456, 605-610 (2008).

64. Naon, D. et al. Critical reappraisal confirms that Mitofusin 2 is an endoplasmic reticulum-mitochondria tether. Proc. Natl Acad. Sci. USA 113 , 11249-11254 (2016).

65. Ainbinder, A., Boncompagni, S., Protasi, F. \& Dirksen, R. T. Role of Mitofusin-2 in mitochondrial localization and calcium uptake in skeletal muscle. Cell Calcium 57, 14-24 (2015).

66. Chen, Y. et al. Mitofusin 2-containing mitochondrial-reticular microdomains direct rapid cardiomyocyte bioenergetic responses via interorganelle $\mathrm{Ca}(2+)$ crosstalk. Circ. Res. 111, 863-875 (2012).

67. Wang, P. T. C. et al. Distinct mechanisms controlling rough and smooth endoplasmic reticulum contacts with mitochondria. J. Cell Sci. 128, 2759-2765 (2015).

68. Leal, N. S. et al. Mitofusin-2 knockdown increases ER-mitochondria contact and decreases amyloid $\beta$-peptide production. J. Cell Mol. Med. 20, 1686-1695 (2016).

69. El Fissi, N. et al. Mitofusin gain and loss of function drive pathogenesis in Drosophila models of CMT2A neuropathy. EMBO Rep. 19, e45241 (2018).

70. Hedskog, L. et al. Modulation of the endoplasmic reticulum-mitochondria interface in Alzheimer's disease and related models. Proc. Natl Acad. Sci. USA 110, 7916-7921 (2013)

\section{Acknowledgements}

We thank J. Yun, M. Guo, and members of the Sheng lab for constructive discussion; K Chamberlain for critical reading and editing; NINDS EM Facility and Light Imaging Facility. The work was supported by the Intramural Research Program of NINDS, NIH ZIA NS003029, and ZIA NS002946 (Z.-H. Sheng) and NINDS Competitive Fellowship (R. Puri).

\section{Author contributions}

R.P. designed the project, performed experiments, and analyzed data; X-T.C performed mitophagy index analysis, M-Y.L. and R.P. performed biochemical analysis; N.H. generated shRNA-resistant Mul1*, Z.-H.S. is the senior author who conceived and directed the project; R.P. and Z.-H.S. wrote the manuscript.

\section{Additional information}

Supplementary Information accompanies this paper at https://doi.org/10.1038/s41467019-11636-5.

Competing interests: The authors declare no competing interests.

Reprints and permission information is available online at http://npg.nature.com/ reprintsandpermissions/

Peer review information: Nature Communications thanks the anonymous reviewers for their contribution to the peer review of this work. Peer reviewer reports are available.

Publisher's note: Springer Nature remains neutral with regard to jurisdictional claims in published maps and institutional affiliations.

Open Access This article is licensed under a Creative Commons Attribution 4.0 International License, which permits use, sharing, adaptation, distribution and reproduction in any medium or format, as long as you give appropriate credit to the original author(s) and the source, provide a link to the Creative Commons license, and indicate if changes were made. The images or other third party material in this article are included in the article's Creative Commons license, unless indicated otherwise in a credit line to the material. If material is not included in the article's Creative Commons license and your intended use is not permitted by statutory regulation or exceeds the permitted use, you will need to obtain permission directly from the copyright holder. To view a copy of this license, visit http://creativecommons.org/ licenses/by/4.0/.

This is a U.S. Government work and not under copyright protection in the US; foreign copyright protection may apply 2019 